\title{
Homogenization of long fiber reinforced composites including fiber bending effects
}

\author{
Poulios, Konstantinos; Niordson, Christian Frithiof
}

Published in:

Journal of the Mechanics and Physics of Solids

Link to article, DOI:

10.1016/j.jmps.2016.04.010

Publication date:

2016

Document Version

Peer reviewed version

Link back to DTU Orbit

Citation (APA):

Poulios, K., \& Niordson, C. F. (2016). Homogenization of long fiber reinforced composites including fiber bending effects. Journal of the Mechanics and Physics of Solids, 94, 433-452. https://doi.org/10.1016/j.jmps.2016.04.010

\section{General rights}

Copyright and moral rights for the publications made accessible in the public portal are retained by the authors and/or other copyright owners and it is a condition of accessing publications that users recognise and abide by the legal requirements associated with these rights.

- Users may download and print one copy of any publication from the public portal for the purpose of private study or research.

- You may not further distribute the material or use it for any profit-making activity or commercial gain

- You may freely distribute the URL identifying the publication in the public portal 


\title{
Homogenization of long fiber reinforced composites including fiber bending effects
}

\author{
Konstantinos Poulios* and Christian F. Niordson ${ }^{\dagger}$ \\ Department of Mechanical Engineering, Technical University of Denmark, Nils Koppels Allé, \\ Building 404, 2800 Kgs. Lyngby, Denmark.
}

April 28, 2016

\begin{abstract}
This paper presents a homogenization method, which accounts for intrinsic size effects related to the fiber diameter in long fiber reinforced composite materials with two independent constitutive models for the matrix and fiber materials. A new choice of internal kinematic variables allows to maintain the kinematics of the two material phases independent from the assumed constitutive models, so that stress-deformation relationships, can be expressed in the framework of hyper-elasticity and hyper-elastoplasticity for the fiber and the matrix materials respectively. The bending stiffness of the reinforcing fibers is captured by higher order strain terms, resulting in an accurate representation of the micro-mechanical behavior of the composite. Numerical examples show that the accuracy of the proposed model is very close to a non-homogenized finite-element model with an explicit discretization of the matrix and the fibers.
\end{abstract}

Keywords: long fiber reinforced composites, strain gradient, anisotropic plasticity, finite-element, large deformation, homogenization.

\section{Introduction}

The extensive use of long fiber reinforced materials, especially in lightweight mechanical constructions, has motivated intensive research efforts in the past decades, especially regarding the understanding of failure mechanisms and the prediction of mechanical strength limits. In particular, the case of failure under compressive loads has received special attention due to its vast practical importance. In this context, both experimental studies and analytical or numerical models, like e.g. in $[1,2,3,4,5,6]$, have contributed to the understanding and quantification of kink band formation and fiber micro-buckling phenomena.

Regarding the numerical modeling of long fiber reinforced composites, there are two main categories of models that can be found in the literature. Individual fiber models rely on an explicit discretization of the matrix and the reinforcing fiber materials by means of a corresponding finite element mesh. The accuracy of this approach, followed for instance in [2, 7, 8], in representing the real micro-mechanical behavior of a composite material is determined rather by the quality of the available material parameters than by any modeling simplifications. However, an explicit discretization of

\footnotetext{
${ }^{*}$ E-mail: kopo@mek.dtu.dk

${ }^{\dagger}$ E-mail: cn@mek.dtu.dk
} 
the fibers is computationally expensive and difficult to apply to complex shaped domains, limiting the applicability of this approach to small sizes and simple geometries. As an alternative, homogenized models can represent the mechanical behavior of composite materials without including the geometry of individual fibers explicitly. For instance, the models presented in [3] and [6] account for the reinforcing fibers by means of appropriate anisotropic material formulations. They are computationally more efficient than individual fiber models and they do not require any intricate mesh generation. This is however at the cost of some simplifications. The model introduced by Fleck and Shu [3] relies on an anisotropic yield function and flow rule, which are rather phenomenological and have to be determined for a specific composite experimentally. Following a different approach, the homogenized model proposed by Christoffersen and Jensen in [6] is based on two independent constitutive laws for the fiber and the matrix materials. However, unlike the former model, the latter neglects the bending stiffness of the fibers.

Apart from numerical models targeting specifically long fiber reinforced composites, generic methods for dealing with anisotropic plasticity can potentially also represent this type of materials as specific case of general anisotropy. Recent extensions of the classical anisotropic yield criterion of Hill [9] to the finite strain regime, can be found, for instance, in [10, 11, 12]. All aforementioned works highlight the necessity of accounting for plastic spin effects in order to track the evolution of preferred directions in the considered materials. Nevertheless, the Hill-type anisotropic yield function and flow rule required by this kind of generic models are phenomenological and have to be obtained through experiments, either on real specimens or by simulations. Moreover, these models are intrinsically limited to a size scale independent material behavior and are consequently not capable of capturing the fiber bending stiffness effect.

In solid mechanics, size scale dependent material behavior is the subject of so-called non-local or higher order theories, like e.g. Cosserat elasticity [13] and strain gradient plasticity. A relatively generic and thermodynamically consistent framework for expressing non-local material behavior is presented e.g. in [14]. Combining this framework with generic anisotopic plasticity models, like the aforementioned [10, 11, 12] would result in a powerful algorithm capable of capturing the behavior of a very wide range of materials including a homogenized representation of long fiber reinforced composites. Nevertheless, the expected complexity of such a generic model should not be underestimated, neither the fact that the description of the material constitutive behavior would still be phenomenological.

As an alternative, the present paper proposes a homogenization method, which captures the anisotropic behavior of long fiber reinforced composites, including the fiber bending stiffness, without relying on complex or phenomenological anisotropic constitutive laws at the level of the homogenized material. The proposed approach is based on a decomposition of the composite kinematics into two components corresponding to the material constituents, which permits the application of standard isotropic hyper-elasticity or hyper-elastoplasticity to each constituent independently. The coupling of the two material phases is done partly at the level of kinematics and partly in the weak formulation of the equilibrium equations. The consistent kinematics description employed, enables to fully understand, at the micro-mechanical level, how high-order terms arise as part of the homogenization procedure, if the fiber bending stiffness is to be taken into account. The paper presents a rigorous derivation of such higher order terms from first principles, purely relying on the micro-mechanical behavior of the composite, avoiding any phenomenological descriptions or ad-hoc terms.

It should be noted that the present work is largely inspired by and combines concepts from [3, 6] and [12]. As in the Cosserat continuum based model presented in [3], the here proposed model relies on the spatial gradient of an internal kinematic variable for capturing bending stiffness effects of the individual layers in the material micro-structure. However, the internal kinematic variable introduced 
in the present work, is a two-dimensional vector instead of a scalar micro-rotation. The cost of the increased dimension of this unknown is justified by the consequent decoupling of the kinematics of the matrix and fiber layers, which makes the formulation rather flexible with respect to material models that can be applied to each constituent individually. Any effort for eliminating the internal kinematic variable or one component of it, by exploiting some information or assumption on the constitutive behavior of the constituents, would compromise the flexibility of the proposed model with respect to imposing independent material models per constituent.

The paper is organized in nine sections. Following this introduction, Section 2 defines all necessary kinematic parameters and Section 3 presents an enhancement of the considered deformation tensors with higher order terms that are necessary for capturing size-scale dependent effects. Section 4 summarizes the multiplicative split of the deformation gradient, which is used for modeling isotropic plasticity within a single constituent. Section 5 presents all assumed constitutive equations for both fiber and matrix materials, while Section 6 derives the virtual work principle equations expressing force and moment equilibrium in the composite. Section 7 presents the final finite-element discretization of these equilibrium equations along with the plastic yield consistency equation. Section 8 is devoted to numerical examples and Section 9 concludes the paper.

\section{Kinematics}

Let $X$ denote a point in a reference configuration of a solid consisting of perfectly bonded matrix material and long reinforcing fibers. If $\hat{\boldsymbol{u}}$ and $\boldsymbol{u}$ denote the actual and the homogenized displacement fields respectively, the location of point $X$ in the deformed solid can be written as $\hat{\boldsymbol{x}}=\boldsymbol{X}+\hat{\boldsymbol{u}}$ for the non-homogenized setting and as $\boldsymbol{x}=\boldsymbol{X}+\boldsymbol{u}$ for the homogenized one. Corresponding deformation gradients can be defined as

$$
\hat{\boldsymbol{F}}=\boldsymbol{I}+\nabla \hat{\boldsymbol{u}}
$$

and

$$
\boldsymbol{F}_{u}=\boldsymbol{I}+\nabla \boldsymbol{u} .
$$

Averaging the deformation gradient $\hat{\boldsymbol{F}}$ over a unit cell volume $V$ of the undeformed periodic microstructure is a homogenization procedure, which is suitable for finite deformations [15]. In the case of the here considered micro-structure such averaging can be performed per constituent volume $V_{m}$ and $V_{f}$ with $V_{m}+V_{f}=V$, as

$$
\frac{1}{V} \int_{V_{m}} \hat{\boldsymbol{F}} d V+\frac{1}{V} \int_{V_{f}} \hat{\boldsymbol{F}} d V=c_{m} \boldsymbol{F}_{m}+c_{f} \boldsymbol{F}_{f},
$$

where $c_{m}$ and $c_{f}$, with $c_{m}+c_{f}=1$, are matrix and fiber volume fractions respectively and the tensors $\boldsymbol{F}_{m}$ and $\boldsymbol{F}_{f}$ are defined as the averages of the deformation gradient $\hat{\boldsymbol{F}}$ within the corresponding constituents.

The considered micro-structure can be embedded in the homogenized setting by equating the homogenized deformation gradient from Eq. (2) to the averaged one from Eq. (3), providing the following rule of mixture

$$
\boldsymbol{F}_{u}=c_{m} \boldsymbol{F}_{m}+c_{f} \boldsymbol{F}_{f} .
$$

Defining the fiber tangent direction in the reference configuration by means of a unit vector $\boldsymbol{T}$ as a function of the considered point $X$, a Voigt-type consistency condition along the fiber direction can be written as

$$
\boldsymbol{F}_{u} \boldsymbol{T}=\boldsymbol{F}_{m} \boldsymbol{T}=\boldsymbol{F}_{f} \boldsymbol{T}
$$


Moreover, with $N$ denoting the fiber normal direction unit vector, it can easily be shown that due to Eq. (4) the homogenized setting provides an unbiased approximation of the actual cross section perpendicular to the fiber direction by verifying the validity of

$$
\boldsymbol{F}_{u}\left(h_{m}+h_{f}\right) \boldsymbol{N}=\boldsymbol{F}_{m} h_{m} \boldsymbol{N}+\boldsymbol{F}_{f} h_{f} \boldsymbol{N},
$$

with $h_{m}$ and $h_{f}$ respectively denoting the matrix and fiber layer heights with $h_{f} / h_{m}=c_{f} / c_{m}$.

At this point a kinematic decomposition is pursued, which consists in finding closed form relationships that express $\boldsymbol{F}_{m}$ and $\boldsymbol{F}_{f}$ as functions of $\boldsymbol{F}_{u}$ and a minimum number of additional necessary kinematic variables. Fig. 1 describes how the initially orthonormal orientation vectors $\boldsymbol{T}$ and $\boldsymbol{N}$ transform within each constituent and the homogenized setting between the reference and some deformed configuration. It also introduces the vector $\boldsymbol{d}$, which accounts for any deviations between the constituents and the homogenized setting, expressed in the deformed configuration. The mathematical definition of $\boldsymbol{d}$ is given below

$$
\boldsymbol{d}=-c_{m}\left(\boldsymbol{F}_{u}-\boldsymbol{F}_{m}\right) N=c_{f}\left(\boldsymbol{F}_{u}-\boldsymbol{F}_{f}\right) \boldsymbol{N},
$$

with the equivalence between the last two alternative expressions originating from Eq. (4) after multiplication from the right side with $N$.
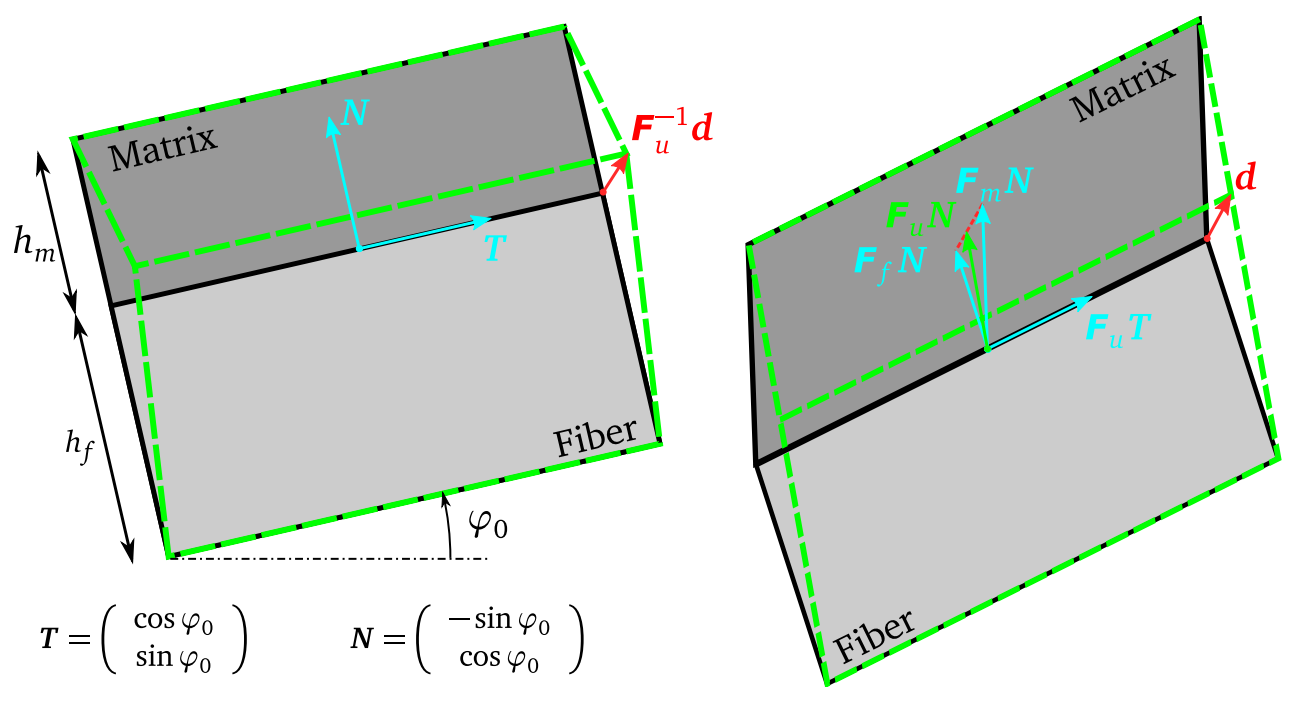

Figure 1: Orientation vectors in the reference (left) and some deformed configuration (right). and $\boldsymbol{F}_{f}$

Combining Eqs. (5) and (7), as detailed in Appendix A, yields the following expressions for $\boldsymbol{F}_{m}$

$$
\boldsymbol{F}_{m}=\boldsymbol{F}_{u}+\frac{1}{c_{m}} \boldsymbol{d} \boldsymbol{N}^{T}
$$

and

$$
\boldsymbol{F}_{f}=\boldsymbol{F}_{u}-\frac{1}{c_{f}} d \boldsymbol{N}^{T}
$$

which complete the intended kinematic decomposition, since the deformation tensors of both constituents are defined as functions of $\boldsymbol{F}_{u}$ and the two components of the additional kinematic variable $\boldsymbol{d}$. Elimination of the latter can be achieved by imposing a Reuss-type equilibrium condition in direction 
perpendicular to the fibers. Such a treatment, although common among many models in the cited literature, has the drawback that it binds the final formulation to specific constitutive models assumed for the two constituents. A main characteristic of the presently proposed method is that it maintains independent kinematic quantities and constitutive models up to the final solution of the global finiteelement system. The aforementioned Reuss-type consistency condition will be enforced within the weak formulation of the global system so that in the final formulation the assumed hyper-elastic and hardening laws for each constituent are easy to replace with alternative ones.

\section{Higher order terms}

The above presented kinematic description of the composite micro-structure, as any similar decomposition not accounting for curvature effects, has one fundamental limitation. It includes no information about the variation of $\boldsymbol{F}_{m}$ or $\boldsymbol{F}_{f}$ over the height of a single layer of matrix or fiber respectively. With regard to Fig. 2, the so far presented kinematics can for example describe the deformed configurations (b) and (c) but they fall short in capturing configurations (d) and (e).

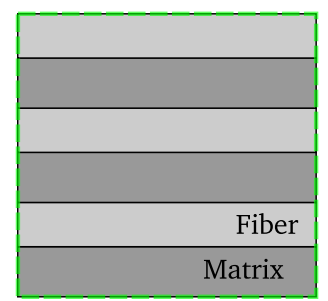

(a)
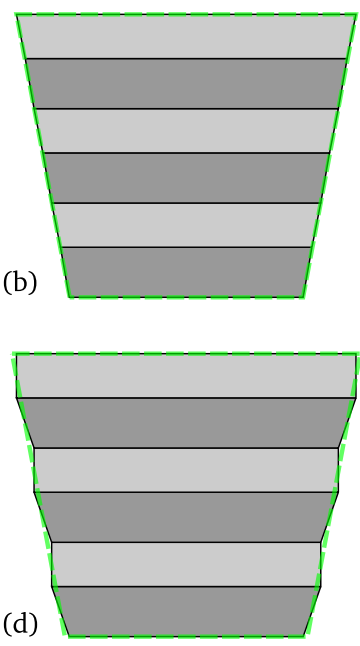

(c)

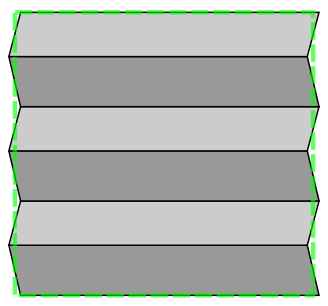

(e)

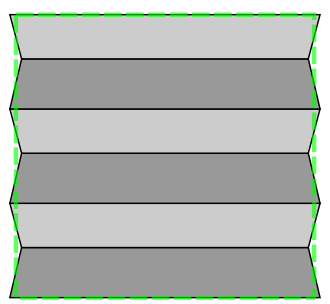

Figure 2: Reference configuration (a) and some possible deformed configurations of the composite microstructure (b,c,d,e).

Configuration (b) corresponds to the trivial case with $\boldsymbol{d}$ equal to zero so that $\boldsymbol{F}_{m}=\boldsymbol{F}_{f}=\boldsymbol{F}_{u}$ and $\boldsymbol{F}_{u}$ itself is sufficient for describing the deformation state over the whole composite consistently. In case (c), there is a non-zero but spatially constant $\boldsymbol{d}$, while $\boldsymbol{F}_{u}$ is assumed for the sake of simplicity to be spatially constant as well. For this configuration $\boldsymbol{F}_{m} \neq \boldsymbol{F}_{f} \neq \boldsymbol{F}_{u}$ but the deformation tensors $\boldsymbol{F}_{m}$ and $\boldsymbol{F}_{f}$ from Eqs. (8) and (9) are sufficient for describing the current deformation state in the complete volume of the matrix and fiber materials respectively.

Although configuration (d) in Fig. 2 is similar to configuration (b) in the sense that $\boldsymbol{d}$ is zero at the center of the cell considered, the gradient of $\boldsymbol{d}$ along the fiber direction is here non-zero. Eqs. (8) and (9) do not differentiate between cases (b) and (d), resulting in the same deformation tensors $\boldsymbol{F}_{m}=\boldsymbol{F}_{f}=\boldsymbol{F}_{u}$ for both cases. This observation demonstrates that Eqs. (8) and (9p) do not capture the actual variation of deformation states across the height of a single matrix or fiber layer. Configuration (e) is a case similar to (d) but with a spatially constant homogenized deformation gradient $\boldsymbol{F}_{u}$. 
An important insight that can be extracted from these examples, is that the proposed kinematic description of Section 2 can capture variations of the deformation state over the height of a single matrix or fiber layer only to the extent that such a variation matches the variation among subsequent layers of the matrix or fiber material. For instance in configuration (d), there is no variation of $\boldsymbol{F}_{f}$ over the fiber height but there is a non-zero variation of $\boldsymbol{F}_{f}$ among the three fibers included in Fig. 2 (d). Some additional treatment is necessary in order to take such cases into account.

The starting point for treating configurations like the ones in Figs. 2(d) and (e) more accurately, is the observation that the deformation tensors defined by Eqs. (8) and (9) do not fulfill an important restriction imposed by the definition of the actual deformation gradient $\hat{\boldsymbol{F}}$ within each individual constituent. According to Eq. (1), noting that the curl of the gradient of a vector field is by definition zero, the deformation gradient $\boldsymbol{F}$ fulfills the following compatibility condition

$$
\frac{\partial \hat{F}_{12}}{\partial X_{1}}-\frac{\partial \hat{F}_{11}}{\partial X_{2}}=0 \quad \text { and } \quad \frac{\partial \hat{F}_{22}}{\partial X_{1}}-\frac{\partial \hat{F}_{21}}{\partial X_{2}}=0
$$

with indexes 1 and 2 referring to the axes of a fixed Cartesian system.

If no connection between the internal kinematic variable $\boldsymbol{d}$ and the underlying non-homogenized displacement field $\hat{\boldsymbol{u}}$ is imposed elsewhere, deformation tensors $\boldsymbol{F}_{m}$ and $\boldsymbol{F}_{f}$ defined through Eqs. (8) and (9) do not necessarily fulfill a compatibility condition in the sense of Eq. (10). This can be demonstrated by e.g. substituting $\hat{\boldsymbol{F}}$ with $\boldsymbol{F}_{f}$ in the left hand side of the first expression in Eq. (10, which then evaluates to

$$
\frac{\partial F_{f 12}}{\partial X_{1}}-\frac{\partial F_{f 11}}{\partial X_{2}}=\frac{\partial F_{u 12}}{\partial X_{1}}-\frac{\partial F_{u 11}}{\partial X_{2}}-\frac{1}{c_{f}}\left(\frac{\partial d_{1}}{\partial X_{1}} N_{2}-\frac{\partial d_{1}}{\partial X_{2}} N_{1}+\left(\frac{\partial N_{2}}{\partial X_{1}}-\frac{\partial N_{1}}{\partial X_{2}}\right) d_{1}\right) \neq 0 .
$$

On the right hand side of Eq. (11), the term depending on $\boldsymbol{F}_{u}$ is zero due to Eq. (2) but the term depending on $\boldsymbol{d}$ and its gradient is in general non-zero for an arbitrary field $\boldsymbol{d}$. Inspired by Eq. (11) and realizing that gradients of $\boldsymbol{d}$ can not at the same time describe changes of the deformation state among neighboring layers and changes through the thickness of an individual layer, a description of the deformation state within a single layer is sought in the form

$$
\tilde{\boldsymbol{F}}_{c}\left(\chi_{N}\right)=\boldsymbol{F}_{c}+\chi_{N} \boldsymbol{A}_{c}, \quad c=m, f,
$$

where $\chi_{N}$ is the signed distance from the centerline of the considered layer, lying in the ranges $\left[-h_{m} / 2, h_{m} / 2\right]$ and $\left[-h_{f} / 2, h_{f} / 2\right]$ for matrix and fiber layers respectively. Then, matrices $\boldsymbol{A}_{m}$ and $\boldsymbol{A}_{f}$ can be defined point-wise so that the enhanced deformation tensors $\tilde{\boldsymbol{F}}_{m}$ and $\tilde{\boldsymbol{F}}_{f}$ fulfill a compatibility condition in the sense of Eq. $(10)$, along the layer centerline $\chi_{N}=0$. As proven in Appendix B, such a treatment leads to $\tilde{\boldsymbol{F}}_{m}$ and $\tilde{\boldsymbol{F}}_{f}$ defined as

$$
\begin{gathered}
\tilde{\boldsymbol{F}}_{m}\left(\chi_{N}\right)=\boldsymbol{F}_{m}+\chi_{N} \frac{1}{c_{m}}\left(\nabla \boldsymbol{d} \boldsymbol{T} \boldsymbol{T}^{T}+\operatorname{div} \boldsymbol{T} \boldsymbol{d} \boldsymbol{T}^{T}\right) \\
\tilde{\boldsymbol{F}}_{f}\left(\chi_{N}\right)=\boldsymbol{F}_{f}-\chi_{N} \frac{1}{c_{f}}\left(\nabla \boldsymbol{d} \boldsymbol{T} \boldsymbol{T}^{T}+\operatorname{div} \boldsymbol{T} \boldsymbol{d} \boldsymbol{T}^{T}\right),
\end{gathered}
$$

which recover the equality in expressions 110 if substituted in place of $\hat{\boldsymbol{F}}$ instead of $\boldsymbol{F}_{m}$ and $\boldsymbol{F}_{f}$. In Eqs. (13) and (14), the scalar quantity $\operatorname{div} \boldsymbol{T}$ is known a priori, resulting from the prescribed field of the tangential unit vector $\boldsymbol{T}$ in the reference configuration. Realistic fiber composites comprise a microstructure of perfectly or approximately parallel layering resulting in $\operatorname{div} \boldsymbol{T}$ being zero or negligible, 
respectively, which leads to a simplification of Eqs. (13) and (14) for a corresponding choice of the field $T$.

In the derivation of force and moment equilibrium equations later, $\chi_{N}$ has to be eliminated by performing analytical integration within the aforementioned intervals. Fig. 3 illustrates how the enhanced deformation tensor $\tilde{\boldsymbol{F}}_{f}$ describes the deformation state at different distances from the centerline of a fiber sample subjected to bending and shearing.
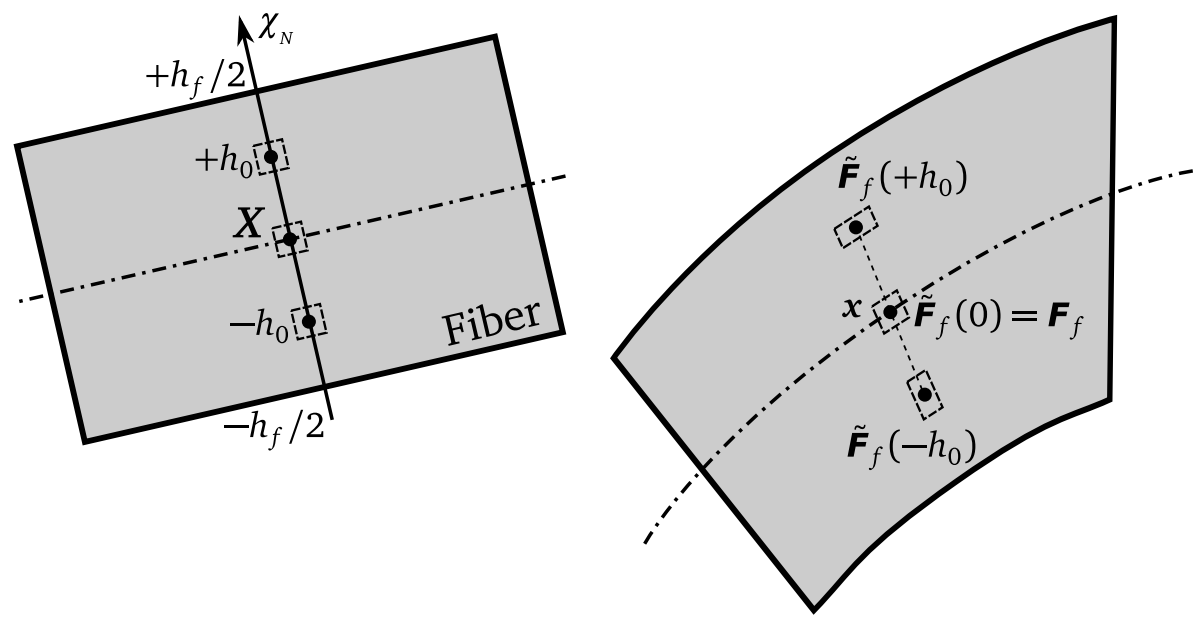

Figure 3: Variation of deformation gradient over the fiber height.

\section{Multiplicative split}

Modeling of isotropic plasticity can be based on the multiplicative split of the deformation gradient tensor into a plastic and an elastic part, as

$$
\boldsymbol{F}=\boldsymbol{F}^{e} \boldsymbol{F}^{p}
$$

with superscripts $e$ and $p$ respectively referring to the elastic and plastic part. Although a corresponding detailed presentation can be found in many literature sources like e.g. [16] and [17], the present section summarizes the most important equations defining a consistent time integration of the aforementioned multiplicative split. Within this section, the index free deformation gradient tensor $\boldsymbol{F}$ will refer to either of $\tilde{\boldsymbol{F}}_{m}$ and $\tilde{\boldsymbol{F}}_{f}$, while all dependent quantities, if not further specified, will respectively refer to either the matrix or the fiber materials.

It is important to note that although all deformation tensors are defined in the previous sections as two-by-two matrices, in the multiplicative split presented here and the constitutive equations presented in the following sections, three-dimensional deformation tensors are required. Without employing any special notation for distinguishing them, three-dimensional versions of deformation tensors, corresponding to $\boldsymbol{F}$ in Eq. (15), are obtained assuming plane-strain deformation conditions by extending the corresponding two-by-two matrices with a row and a column filled with zero off-diagonal elements and the unit as the third diagonal element. In the formalism of this and the subsequent sections, matrices $\boldsymbol{F}^{e}$ and $\boldsymbol{F}^{p}$ as well as all thereupon dependent matrices are three-dimensional. The following presentation is kept generic three-dimensional, although an actual two-dimensional plane- 
strain implementation can exploit the fact that elements $(1,3),(2,3),(3,1)$ and $(3,2)$ of matrices $\boldsymbol{F}^{e}$ and $\boldsymbol{F}^{p}$ are zero.

In finite strain isotropic plasticity, given the plastic part $\boldsymbol{F}_{*}^{p}$ of the deformation gradient, with subscript " $*$ " indicating the previous time instant, the corresponding right Cauchy-Green strain tensor is defined as

$$
\boldsymbol{C}_{*}^{p}=\boldsymbol{F}_{*}^{p T} \boldsymbol{F}_{*}^{p} .
$$

An exponential map based time integration step consists in finding the elastic left Cauchy-Green strain tensor

$$
\boldsymbol{b}^{e}=\boldsymbol{F}^{e} \boldsymbol{F}^{e T}
$$

at the current step, based on a trial elastic step defined as

$$
\boldsymbol{b}^{e, t r i a l}=\boldsymbol{F}\left(\boldsymbol{C}_{*}^{p}\right)^{-1} \boldsymbol{F}^{T}
$$

As detailed in [17], the final equation accounting for the plastic flow over the considered time step is

$$
\ln \boldsymbol{b}^{e}=\ln \boldsymbol{b}^{e, t r i a l}-2 \boldsymbol{\Delta} \boldsymbol{\epsilon}^{p},
$$

with $\boldsymbol{\Delta} \boldsymbol{\epsilon}^{p}$ being a symmetric tensor corresponding to the plastic strain increment during the considered time step. Its direction has to be specified according to some plastic flow rule, while its magnitude is linked to a plastic hardening law.

Under consideration of definitions (16), (17) and (18), Eq. (19) provides a closed form expression for the elastic strain tensor $\boldsymbol{b}^{e}$ as a function of a plastic strain at the previous time instant, $\boldsymbol{C}_{*}^{p}$, and the, yet to be determined, total strain $\boldsymbol{F}$ and plastic strain increment $\boldsymbol{\Delta} \boldsymbol{\epsilon}^{p}$.

Based on Eqs. (16)-(19), it is possible to derive an update equation for $\boldsymbol{C}^{p}$ as

$$
\left(\boldsymbol{C}^{p}\right)^{-1}=\left(\boldsymbol{F}^{-1} \exp \left(-\boldsymbol{\Delta} \boldsymbol{\epsilon}^{p}\right) \boldsymbol{F}\right)\left(\boldsymbol{C}_{*}^{p}\right)^{-1}\left(\boldsymbol{F}^{-1} \exp \left(-\boldsymbol{\Delta} \boldsymbol{\epsilon}^{p}\right) \boldsymbol{F}\right)^{T} .
$$

Note that in an actual implementation of this algorithm, there is no need for evaluating and storing $\boldsymbol{C}^{p}$. It is only necessary to evaluate $\left(\boldsymbol{C}^{p}\right)^{-1}$ directly and store its components.

\section{Constitutive equations}

In order to complete the problem setting, constitutive equations are required in the form of an elastic strain-stress relationship, a plastic yield criterion and a plastic flow rule for each constituent.

Requiring that the reference configuration assumed in the previous kinematic analysis corresponds to an undeformed state free of tractions on the boundaries and residual stresses in the interior of the considered solid, each constituent can be defined in the framework of hyper-elasticity by means of an elastic free energy density function in terms of the elastic left Cauchy-Green strain tensor. A common free energy density function corresponding to a Neo-Hookean material law is the following

$$
\psi^{e}\left(\boldsymbol{b}^{e}\right)=\frac{K}{8}\left(\ln \left|\boldsymbol{b}^{e}\right|\right)^{2}+\frac{G}{2}\left(\operatorname{Tr}\left(\left|\boldsymbol{b}^{e}\right|^{-1 / 3} \boldsymbol{b}^{e}\right)-3\right) .
$$

More convenient for being incorporated to the modeling of plastic deformation is the logarithmic strain based Hencky material model which is defined through

$$
\psi^{e}\left(\boldsymbol{b}^{e}\right)=\frac{K}{8}\left(\ln \left|\boldsymbol{b}^{e}\right|\right)^{2}+\frac{G}{4}\left\|\operatorname{dev}\left(\ln \boldsymbol{b}^{e}\right)\right\|^{2},
$$


with material parameters $K$ and $G$ being the bulk and shear moduli, explicitly written as $K_{m}$, $G_{m}$ and $K_{f}, G_{f}$ for the matrix and the fibers respectively. The following relation regarding the determinant $\left|\boldsymbol{b}^{e}\right|$ can be noted

$$
\left|\boldsymbol{b}^{e}\right|^{1 / 2}=\left|\boldsymbol{F}^{e}\right|=|\boldsymbol{F}|,
$$

where the last equality is based on isochoric plastic deformation.

Based on the free energy density functions defined in Eqs. (21) and (22) and making use of Eq. (23), the corresponding Kirchhoff stresses are given by

$$
\boldsymbol{\tau}=K \ln |\boldsymbol{F}| \boldsymbol{I}+G|\boldsymbol{F}|^{-2 / 3} \operatorname{dev}\left(\boldsymbol{b}^{e}\right)
$$

and

$$
\boldsymbol{\tau}=K \ln |\boldsymbol{F}| \boldsymbol{I}+G|\boldsymbol{F}|^{-2 / 3} \operatorname{dev}\left(\ln \boldsymbol{b}^{e}\right),
$$

respectively.

Some further stress tensors which will be of use later are the Cauchy stress tensor

$$
\boldsymbol{\sigma}=\boldsymbol{\tau} /|\boldsymbol{F}|
$$

and the first Piola-Kirchhoff stress tensor

$$
\boldsymbol{P}=\boldsymbol{\tau} \boldsymbol{F}^{-T}
$$

It should be noted that these stress quantities depend through Eq. (19) on the unknown plastic strain increment during the current time step, $\boldsymbol{\Delta} \boldsymbol{\epsilon}^{p}$. A yield function and a plastic flow rule are required in order to determine the magnitude and direction of $\boldsymbol{\Delta} \boldsymbol{\epsilon}^{p}$ respectively.

In case of isotropic plasticity with linear hardening the set of admissible Cauchy stresses is defined by the following inequality

$$
\|\operatorname{dev}(\boldsymbol{\sigma})\|-\sqrt{2 / 3}\left(\sigma_{y}+H \sqrt{2 / 3} \gamma\right) \leq 0
$$

with $\gamma$ being a scalar measure of accumulated plastic strain. The $\sqrt{2 / 3}$ factors in the last expression are necessary because the initial yield limit $\sigma_{y}$ and hardening modulus $H$ refer to Von Mises stress and equivalent strain values as they are typically obtained in uniaxial tension tests. The assumed linear hardening relationship is chosen only as an example and can easily be replaced through more sophisticated hardening laws.

Based on the following definition of the accumulated plastic strain

$$
\gamma(t)=\int_{0}^{t}\left\|\dot{\boldsymbol{\epsilon}}^{p}\right\| \mathrm{d} t,
$$

the increase from the previous time instant $t_{*}$ to the current instant $t$ can be approximated as

$$
\Delta \gamma=\gamma-\gamma_{*} \approx\left\|\boldsymbol{\Delta} \boldsymbol{\epsilon}^{p}\right\|
$$

In the limit where the plastic flow rate $\dot{\boldsymbol{\epsilon}}^{p}$ remaining co-directional to $\boldsymbol{\Delta} \boldsymbol{\epsilon}^{p}$ during the complete interval from $t_{*}$ to $t$, the approximate Eq. (30) yields an exact one.

Regarding the plastic flow rule, associative plasticity suggests that the plastic strain increment $\boldsymbol{\Delta} \boldsymbol{\epsilon}^{p}$ is parallel to the deviatoric part of the Kirchhoff stress tensor $\boldsymbol{\tau}$, so that

$$
\frac{\boldsymbol{\Delta} \boldsymbol{\epsilon}^{p}}{\left\|\boldsymbol{\Delta} \boldsymbol{\epsilon}^{p}\right\|}=\frac{\operatorname{dev}(\boldsymbol{\tau})}{\|\operatorname{dev}(\boldsymbol{\tau})\|},
$$


where $\boldsymbol{\tau}$ can be considered e.g. at the beginning or the end of the current time step, depending on the intended time integration scheme.

For the Hencky constitutive law, expressed through Eq. (25), it can be proven (see Appendix C) that

$$
\frac{\boldsymbol{\Delta} \boldsymbol{\epsilon}^{p}}{\left\|\boldsymbol{\Delta} \boldsymbol{\epsilon}^{p}\right\|}=\frac{\operatorname{dev}\left(\ln \boldsymbol{b}^{e, t r i a l}\right)}{\left\|\operatorname{dev}\left(\ln \boldsymbol{b}^{e, t r i a l}\right)\right\|},
$$

so that it is possible to write

$$
\boldsymbol{\Delta} \boldsymbol{\epsilon}^{p}=\xi \operatorname{dev}\left(\ln \boldsymbol{b}^{e, t r i a l}\right)
$$

with the coefficient $\xi$ defined as

$$
\xi=\frac{\Delta \gamma}{\left\|\operatorname{dev}\left(\ln \boldsymbol{b}^{e, t r i a l}\right)\right\|} .
$$

Substituting Eq. (33) into $\boldsymbol{\sigma}$ and $\gamma$ in Eq. (28) allows to solve for $\xi$, so that $\xi$ and consequently also $\boldsymbol{\Delta} \boldsymbol{\epsilon}^{p}$ could be considered as implicit functions of $\boldsymbol{u}, \boldsymbol{d}$ and the state variable $\left(\boldsymbol{C}_{*}^{p}\right)^{-1}$. This is a very common procedure in the modeling of plasticity which nevertheless has a practical drawback. In order to achieve proper linearization of $\boldsymbol{\Delta} \boldsymbol{\epsilon}^{p}$ and any dependent quantity, implicit differentiation based on Eq. (28) is required, which adds complexity to the implementation of the algorithm and makes such an implementation dependent on an assumed hardening law. In the present work, a less common but more generic approach will be followed by considering $\xi$ as an additional unknown that will be approximated on an appropriate finite-element space. As it will be described in Section 7. Eq. (28) is finally incorporated in the discretized global system.

\section{Virtual work principle}

Let domain $\Omega_{0} \subset \mathbb{R}^{2}$, shown in Fig. 4, describe the undeformed geometry of a two-dimensional construction made of the considered long fiber reinforced composite material. With point $X \in \Omega_{0}$ denoting an arbitrary location in this undeformed configuration, the orientation angle and volumetric content of the reinforcing fibers can be considered as spatial functions $\varphi_{0}(X)$ and $c_{f}(X)$ respectively. The dashed lines in Fig. 4 illustrate the fiber orientation and their density represents the fiber content.

The problem setting is complemented by a given time history of boundary conditions applied to the domain boundary $\Gamma_{0}$. Such boundary conditions can actually be prescribed kinematic variables, as on the portion $\Gamma_{0 a} \cup \Gamma_{0 b}$ of the domain boundary in Fig. 4, or corresponding tractions. Neglecting inertial and viscous forces the response of the considered structure to the imposed boundary conditions can be considered as a quasi-static procedure. Force equilibrium with respect to a deformed configuration $\Omega$ at any arbitrary time instant can be expressed through the principle of virtual work, resulting in a weak formulation of the problem in hand, appropriate for discretization.

Considering the displacements field $\boldsymbol{u}$ and the quantity $\boldsymbol{d}$ as independent kinematic variables, corresponding fictitious variations can be denoted with $\boldsymbol{\delta} \boldsymbol{u}$ and $\boldsymbol{\delta} \boldsymbol{d}$. Based on the work-conjugacy between the Kirchhoff stress and the spatial velocity gradient, the virtual work variation in each constituent can be expressed as

$$
\delta \tilde{W}_{c}=\tilde{\boldsymbol{\tau}}_{c}:\left(\boldsymbol{\delta} \tilde{\boldsymbol{F}}_{c} \tilde{\boldsymbol{F}}_{c}^{-1}\right), c=m, f
$$

where the tilde symbol refers to quantities based on the enhanced deformation tensors from Eqs. (13) and (14). The colon symbol denotes the Frobenius product of two matrices $\boldsymbol{A}$ and $\boldsymbol{B}$, defined as 


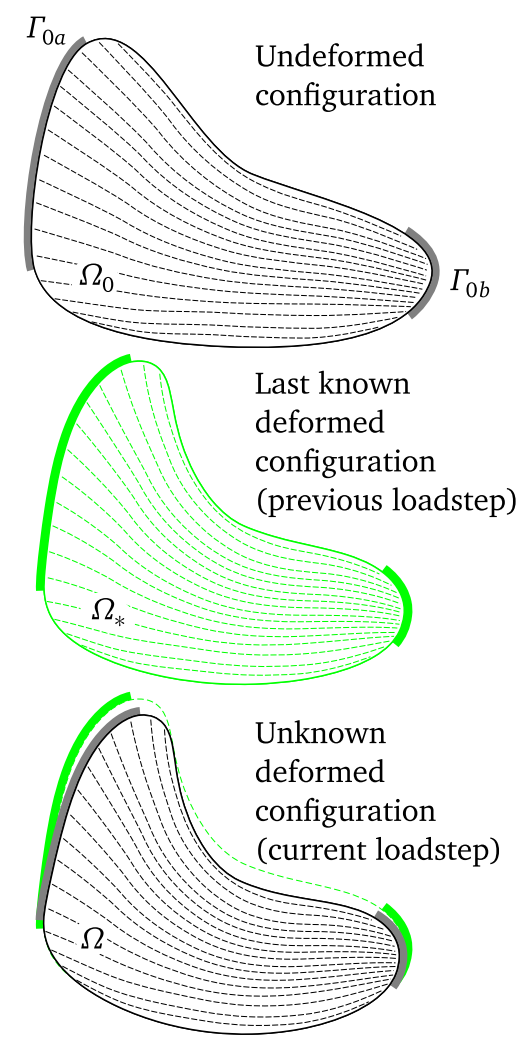

Figure 4: Geometry and boundary conditions in the undeformed and deformed configurations.

$\boldsymbol{A}: \boldsymbol{B}=\operatorname{Tr}\left(\boldsymbol{A}^{T} \boldsymbol{B}\right)$. Based on the first Piola-Kirchhoff stress tensor defined in Eq (27), Eq. (35) can be rewritten in the equivalent form

$$
\delta \tilde{W}_{c}=\tilde{\boldsymbol{P}}_{c}: \boldsymbol{\delta} \tilde{\boldsymbol{F}}_{c}, \quad c=m, f .
$$

Note that the here considered virtual work expresses energy density with respect to the undeformed volume, while it includes both stored energy due to elastic deformation and dissipated energy due to plastic yielding. Also note that under the plane-strain deformation assumption, all matrices involved in the calculation of virtual work quantities are of size two-by-two. This means that stress tensors substituted e.g. from Eqs. (24), (25) or (27) are silently converted from three to two dimensions by simply dropping the third column and row.

Based on the above, one can also define the following virtual work variations, averaged over the height of the considered layer, as

$$
\delta W_{c}=\frac{1}{h_{c}} \int_{-h_{c} / 2}^{h_{c} / 2} \delta \tilde{W}_{c}\left(\chi_{N}\right) d \chi_{N} \quad, \quad c=m, f .
$$

Subsequently, the total virtual work variation per unit volume of the undeformed composite is

$$
\delta W=c_{m} \delta W_{m}+c_{f} \delta W_{f} .
$$

Although it is theoretically possible to account for the multiplicative split from Section 4 in the analytical integration of Eq. (37), the presentation in the current paper will, in the following, be restricted 
to a special case. Assuming that plastic deformation can only take place within the matrix material and that higher order terms become significant only for the reinforcing fibers, intricate mathematical derivations can be avoided.

Under these assumptions, the virtual work variation in the matrix can be written as

$$
\delta W_{m}=\boldsymbol{\tau}_{m}\left(\boldsymbol{b}_{m}^{e}\right):\left(\boldsymbol{\delta} \boldsymbol{F}_{m} \boldsymbol{F}_{m}^{-1}\right),
$$

with $\boldsymbol{\tau}_{m}$ according to the constitutive law Eq. (25), $\boldsymbol{b}_{m}^{e}$ fulfilling Eq. (19), $\boldsymbol{F}_{m}$ given by Eq. (8) and $\boldsymbol{\delta}_{m}$ evaluated, based on Eqs. (2) and (8), as

$$
\boldsymbol{\delta} \boldsymbol{F}_{m}=\nabla \boldsymbol{\delta} \boldsymbol{u}+\frac{1}{c_{m}} \boldsymbol{\delta} \boldsymbol{d} \boldsymbol{N}^{T}
$$

In the treatment of the fiber layers, the rather unusual case of $\operatorname{div} \boldsymbol{T} \neq 0$ will not be included in the following derivations, for the sake of simplicity. Hence, the enhanced deformation tensor $\tilde{\boldsymbol{F}}_{f}$ from Eq. (14) has the simplified form

$$
\tilde{\boldsymbol{F}}_{f}\left(\chi_{N}\right)=\boldsymbol{F}_{f}-\chi_{N} \frac{1}{c_{f}} \nabla \boldsymbol{d} \boldsymbol{T} \boldsymbol{T}^{T}
$$

The variations of the original and the enhanced deformation tensors in the fiber material from Eqs. (9) and (41), are respectively defined as

$$
\boldsymbol{\delta} \boldsymbol{F}_{f}=\nabla \boldsymbol{\delta} u-\frac{1}{c_{f}} \delta \boldsymbol{d} N^{T}
$$

and

$$
\boldsymbol{\delta} \tilde{\boldsymbol{F}}_{f}=\boldsymbol{\delta} \boldsymbol{F}_{f}-\chi_{N} \frac{1}{c_{f}} \nabla \boldsymbol{\delta} \boldsymbol{d} \boldsymbol{T} \boldsymbol{T}^{T} .
$$

It should be noted that for purely elastic behavior of the fibers, the first Piola-Kirchhoff stress in Eq. (36) is a function of the enhanced deformation tensor from Eq. (41), i.e.

$$
\tilde{\boldsymbol{P}}_{f}\left(\tilde{\boldsymbol{F}}_{f}\right)=\tilde{\boldsymbol{P}}_{f}\left(\boldsymbol{F}_{f}, \chi_{N} \nabla \boldsymbol{d} T \boldsymbol{T}^{T}\right) .
$$

In order to simplify the analytical integration in Eq. (37), one can linearize $\tilde{\boldsymbol{P}}_{f}$ with respect to the higher order term as

$$
\tilde{\boldsymbol{P}}_{f} \approx \boldsymbol{P}_{f}-\chi_{N} \frac{1}{c_{f}} \mathcal{D} \boldsymbol{P}_{f}\left(\boldsymbol{F}_{f}\right)\left[\nabla \boldsymbol{d} \boldsymbol{T} \boldsymbol{T}^{T}\right]
$$

where $\boldsymbol{P}_{f}$ is the first Piola-Kirchhoff stress, based on the non-enhanced deformation tensor, and $\mathcal{D} \boldsymbol{P}_{f}\left(\boldsymbol{F}_{f}\right)[\boldsymbol{\Theta}]$ its directional derivative in direction $\boldsymbol{\Theta}$, obtained through differentiation of $\boldsymbol{P}_{f}$ based on the constitutive law Eq. (24), as

$$
\begin{aligned}
\mathcal{D} \boldsymbol{P}_{f}\left(\boldsymbol{F}_{f}\right)[\boldsymbol{\Theta}]= & \left(\boldsymbol{F}_{f}^{-T}: \boldsymbol{\Theta}\right)\left(K_{f} \boldsymbol{I}-\frac{2 G_{f}}{3\left|F_{f}\right|^{2 / 3}} \operatorname{dev}\left(\boldsymbol{F}_{f} \boldsymbol{F}_{f}^{T}\right)\right) \boldsymbol{F}_{f}^{-T} \\
& +\boldsymbol{P}_{f} \boldsymbol{\Theta} \boldsymbol{F}_{f}^{-T}+\frac{G_{f}}{\left|F_{f}\right|^{2 / 3}} \operatorname{dev}\left(\boldsymbol{\Theta} \boldsymbol{F}_{f}^{T}+\boldsymbol{F}_{f} \boldsymbol{\Theta}^{T}\right) \boldsymbol{F}_{f}^{-T} .
\end{aligned}
$$

Substitution of Eqs. (43) and (45) into Eq. (36) results in linear, quadratic and constant terms with respect to $\chi_{N}$. Due to the symmetric limits of the integral in Eq. (37) all linear terms evaluate 
to zero, hence only constant and quadratic terms are to be considered. Finally, the averaged virtual work variation in the fibers evaluates to

$$
\delta W_{f}=\boldsymbol{\tau}_{f}:\left(\boldsymbol{\delta} \boldsymbol{F}_{f} \boldsymbol{F}_{f}^{-1}\right)+\frac{h_{f}^{2}}{12 c_{f}^{2}} \mathcal{D} \boldsymbol{P}_{f}\left(\boldsymbol{F}_{f}\right)\left[\nabla \boldsymbol{d} \boldsymbol{T} \boldsymbol{T}^{T}\right]:\left(\nabla \boldsymbol{\delta} \boldsymbol{d} \boldsymbol{T} \boldsymbol{T}^{T}\right) .
$$

Combining Eqs. (39), (40), 42), (43) and (47) with the total virtual work definition of Eq. (38), yields

$$
\begin{aligned}
\delta W= & c_{m} \boldsymbol{\tau}_{m}:\left(\nabla \boldsymbol{\delta} \boldsymbol{u} \boldsymbol{F}_{m}^{-1}\right)+c_{f} \boldsymbol{\tau}_{f}:\left(\nabla \boldsymbol{\delta} \boldsymbol{u} \boldsymbol{F}_{f}^{-1}\right) \\
& +\boldsymbol{\tau}_{m}:\left(\boldsymbol{\delta} \boldsymbol{d} \boldsymbol{N}^{T} \boldsymbol{F}_{m}^{-1}\right)-\boldsymbol{\tau}_{f}:\left(\boldsymbol{\delta} \boldsymbol{d} \boldsymbol{N}^{T} \boldsymbol{F}_{f}^{-1}\right) \\
& +\frac{h_{f}^{2}}{12 c_{f}} \mathcal{D} \boldsymbol{P}_{f}\left(\boldsymbol{F}_{f}\right)\left[\nabla \boldsymbol{d} \boldsymbol{T} \boldsymbol{T}^{T}\right]:\left(\nabla \boldsymbol{\delta} \boldsymbol{d} \boldsymbol{T} \boldsymbol{T}^{T}\right) .
\end{aligned}
$$

Note that the two terms in the second row of Eq. (48) correspond to the enforcement of the Reusstype equilibrium condition between the fibers and the matrix, in direction perpendicular to the fibers. The higher order term in the third row of Eq. (48) represents the portion of the bending stiffness of the reinforcing fibers, which is not captured by the homogenized displacements field $\boldsymbol{u}$. Through $h_{f}$, appearing in the last term, the final model is size-scale dependent.

Finally, force equilibrium can be expressed through the following weak formulation

$$
\int_{\Omega_{0}} \delta W\left(\boldsymbol{u}, \boldsymbol{d}, \xi_{m}, \boldsymbol{\delta} \boldsymbol{u}, \boldsymbol{\delta} \boldsymbol{d}\right) d X+\int_{\Gamma_{0}} \boldsymbol{f}_{u} \cdot \boldsymbol{\delta} \boldsymbol{u}+\boldsymbol{f}_{d} \cdot \boldsymbol{\delta} \boldsymbol{d} d X=0 \quad \forall \boldsymbol{\delta} \boldsymbol{u}, \boldsymbol{\delta} \boldsymbol{d},
$$

where $f_{u}$ and $f_{d}$ are surface loads respectively work-conjugate to the kinematic variables $\boldsymbol{u}$ and $\boldsymbol{d}$. The load $f_{u}$, which is normally the one prescribed as an input, is a vector field describing a direction with respect to the current configuration $\Omega$, while its magnitude expresses a force density with respect to surface areas defined in the undeformed configuration $\Omega_{0}$. Instead of specifying $f_{u}$ and $f_{d}$ directly, these quantities can also be replaced through corresponding Lagrange multipliers arising from the enforcement of displacement based boundary conditions at portion of $\Gamma_{0}$.

\section{Finite-element discretization}

In order to determine the response of the considered mechanical system numerically, both spatial and time discretizations are necessary. In the present work, 8-node second order quadrilateral elements are used for the discretization of the geometry and a classical backward Euler scheme is employed for discretizing the evolution of time dependent quantities in a total Lagrangian formulation.

In conjunction with the discretization of the geometry, all relevant unknown field quantities are discretized as well, not necessarily with the same order of approximation. The main unknowns of the problem setting, as deployed in the previous sections, consist of the displacements $u$, the internal kinematic variable $\boldsymbol{d}$ and the plastic flow in the matrix coefficient $\xi_{m}$. In the present work, $\boldsymbol{u}$ and $\xi_{m}$ are approximated by 8-node quadrilateral elements, while both possibilities of 4-node linear and 8-node quadrilateral elements are examined with respect to $\boldsymbol{d}$.

Here it should be noted that the last known configuration $\Omega_{*}$ at time $t_{*}$ is defined in the discretized setting through the values of the inverse plastic strain tensor $\left(\boldsymbol{c}_{m *}^{p}\right)^{-1}$ and the accumulated plastic strain measure $\gamma_{m *}$ in the matrix phase, which are stored integration-point-wise. Calculated field 
data $\boldsymbol{u}_{*}, \boldsymbol{d}_{*}$ and $\xi_{m *}$ at the previous time instant are not strictly necessary for solving the current time step, but the first two of them are used as initial guesses for the corresponding unknowns at the current time instant, while a zero initial guess is used with respect to the third unknown $\xi_{m}$.

In order to solve for the three unknowns $\boldsymbol{u}, \boldsymbol{d}$ and $\xi_{m}$, two equations are necessary:

1. Equilibrium between the internal virtual work and the virtual work corresponding to external loads and boundary conditions, see Eq. (49).

2. Fulfillment of the yield criterion corresponding to equality in expression (28) or $\xi_{m}=0$ for negative values of the yield function.

A discrete version of Eq. (49) is obtained by replacing $\boldsymbol{u}, \boldsymbol{d}$ and $\xi_{m}$ with the corresponding finiteelement approximated fields $\boldsymbol{u}^{h}, \boldsymbol{d}^{h}$ and $\xi_{m}^{h}$ and the virtual variations $\boldsymbol{\delta} \boldsymbol{u}$ and $\boldsymbol{\delta} \boldsymbol{d}$ with a finite number of trial variations $\boldsymbol{\delta} \boldsymbol{u}^{h}$ and $\boldsymbol{\delta} \boldsymbol{d}^{h}$ corresponding to the basis functions for the individual degrees of freedom of the assumed finite-element approximations. Additionally, the resulting discrete nonlinear equation needs to be linearized in order to apply Newton's algorithm. If $\boldsymbol{u}^{h(k)}, \boldsymbol{d}^{h(k)}$ and $\xi_{m}^{h(k)}$ represent the $k^{\text {th }}$ step of Newton's algorithm and $\Delta \boldsymbol{u}^{h}, \boldsymbol{\Delta} \boldsymbol{d}^{h}$ and $\Delta \xi_{m}^{h}$ are the yet to be defined increments from the $k^{\text {th }}$ to the $(k+1)^{\text {th }}$ step, a discretized and linearized version of Eq. (49) reads

$$
\begin{aligned}
& \int_{\Omega_{0}}\left(\boldsymbol{\delta} W_{u}^{(k)} \cdot \Delta \boldsymbol{u}^{h}+\boldsymbol{\delta} \boldsymbol{W}_{d}^{(k)} \cdot \boldsymbol{\Delta} \boldsymbol{d}^{h}+\delta W_{\xi_{m}}^{(k)} \Delta \xi_{m}^{h}\right) d X \\
&=-\int_{\Omega_{0}} \delta W^{(k)} d X-\int_{\Gamma_{0}}\left(\boldsymbol{f}_{u} \cdot \boldsymbol{\delta} \boldsymbol{u}+\boldsymbol{f}_{d} \cdot \boldsymbol{\delta} \boldsymbol{d}\right) d X \quad \forall \boldsymbol{\delta} \boldsymbol{u}^{h}, \boldsymbol{\delta} \boldsymbol{d}^{h},
\end{aligned}
$$

where $\delta W_{u}^{(k)}, \delta W_{d}^{(k)}$ and $\delta W_{\xi_{m}}^{(k)}$ are derivatives of Eq. 48 with respect to $\boldsymbol{u}, \boldsymbol{d}$ and $\xi_{m}$ at point $\left(\boldsymbol{u}^{h(k)}, \boldsymbol{d}^{h(k)}, \xi_{m}^{h(k)}\right)$.

The second of the aforementioned equations, namely the one that expresses the plastic yield criterion, can be formulated as a minimization of the following error function

$$
r\left(\boldsymbol{u}, \boldsymbol{d}, \boldsymbol{\xi}_{m}\right)=\left\|\operatorname{dev}\left(\boldsymbol{\sigma}_{m}\right)\right\|-\min \left(\sqrt{2 / 3}\left(\sigma_{y m}+H_{m} \sqrt{2 / 3} \gamma_{m}\right),\left\|\operatorname{dev}\left(\boldsymbol{\sigma}_{m}^{\text {trial }}\right)\right\|\right),
$$

where, according to Eqs. (30) and (34),

$$
\gamma_{m}=\gamma_{m *}+\xi_{m}\left\|\operatorname{dev}\left(\ln \boldsymbol{b}_{m}^{e, t \text { trial }}\right)\right\|
$$

and the trial Cauchy stress tensor of the matrix phase is simply defined as

$$
\boldsymbol{\sigma}_{m}^{\text {trial }}(\boldsymbol{u}, \boldsymbol{d})=\boldsymbol{\sigma}_{m}\left(\boldsymbol{u}, \boldsymbol{d}, \xi_{m}=0\right) \text {. }
$$

Following the Galerkin discretization procedure, a discretized weak form is obtained as

$$
\int_{\Omega_{0}} r\left(\boldsymbol{u}^{h}, \boldsymbol{d}^{h}, \xi_{m}^{h}\right) \delta \xi^{h} d X=0 \quad \forall \delta \xi^{h}
$$

with the trial variations $\delta \xi^{h}$ representing the basis functions used for the finite-element approximation $\xi_{m}^{h}$ of $\xi_{m}$. A linearized version of Eq. 54 , expressing the $(k+1)^{\text {th }}$ Newton's iteration step, reads

$$
\int_{\Omega_{0}}\left(\boldsymbol{r}_{u}^{(k)} \cdot \Delta \boldsymbol{u}^{h}+\boldsymbol{r}_{d}^{(k)} \cdot \Delta \boldsymbol{d}^{h}+r_{\xi_{m}}^{(k)} \Delta \xi_{m}^{h}\right) \delta \xi^{h} d X=-\int_{\Omega_{0}} r^{(k)} \delta \xi^{h} d X \quad \forall \delta \xi^{h},
$$


where $\boldsymbol{r}_{u}^{(k)}, \boldsymbol{r}_{d}^{(k)}$ and $r_{\xi_{m}}^{(k)}$ are derivatives of Eq. 51 with respect to $\boldsymbol{u}, \boldsymbol{d}$ and $\xi_{m}$ and $r^{(k)}$ is the value of $r$, at point $\left(\boldsymbol{u}^{h(k)}, \boldsymbol{d}^{h(k)}, \xi_{m}^{h(k)}\right)$.

After solving the linear system of equations (50) and (55) for $\Delta \boldsymbol{u}^{h}, \boldsymbol{\Delta} \boldsymbol{d}^{h}$ and $\Delta \xi_{m}^{h}$, the $(k+1)^{\text {th }}$ estimate in Newton's algorithm is

$$
\begin{aligned}
& \boldsymbol{u}^{h(k+1)}=\boldsymbol{u}^{h(k)}+\Delta \boldsymbol{u}^{h} \\
& \boldsymbol{d}^{h(k+1)}=\boldsymbol{d}^{h(k)}+\Delta \boldsymbol{d}^{h} \\
& \xi_{m}^{h(k+1)}=\xi_{m}^{h(k)}+\Delta \xi_{m}^{h} .
\end{aligned}
$$

Convergence of Newton's algorithm leads to the final solution of the current time step. Solution of the next time step is then possible after updating $\left(\boldsymbol{c}_{m *}^{p}\right)^{-1}$ and $\gamma_{m *}$ at each integration point with the values calculated according to Eqs. (20) and (52) as well as the boundary conditions with the values corresponding to the next time instant.

Regarding the derivatives $\delta \boldsymbol{W}_{u}, \boldsymbol{\delta} \boldsymbol{W}_{d}, \delta W_{\xi_{m}}, \boldsymbol{r}_{u}, \boldsymbol{r}_{d}$ and $r_{\xi_{m}}$, which are required for assembling the linearized system of Eqs. (50) and (55), differentiation of $\delta W$ and $r$ from Eqs. (48) and (51) respectively is a diligent but trivial procedure. The only exception is the differentiation of the matrix logarithm operator which is in general not straightforward. In our implementation, the matrix logarithm derivative is evaluated as the inverse of the corresponding derivative of the exponential matrix operator, which in turn is evaluated according to the Taylor expansion based method presented in [18]. For the calculation of the matrix logarithm itself, the method documented in [19, Ch. 11] has been used. Our implementation of the proposed model is based on the finite-element framework GetFEM++, [20], which incorporates symbolic differentiation functionality, so that it was not necessary to obtain and implement expressions for $\delta W_{u}, \delta W_{d}, \delta W_{\xi_{m}}, \boldsymbol{r}_{u}, \boldsymbol{r}_{d}$ and $r_{\xi_{m}}$ by hand.

\section{Numerical examples}

This section presents some numerical examples that show how the proposed homogenization performs compared to an explicit discretization of the composite micro-structure with finite elements. Unless otherwise specified, discretization in the presented examples is based on 8-node quadrilateral elements and Gauss-Legendre quadrature with 9 integration points per element is employed for numerical integration.

\subsection{Shearing transverse to the fiber direction}

Fig. 5 shows a unit-cell consisting of a single reinforcing fiber embedded in the matrix material. The length of the cell is considered constant and equal to $1 \mathrm{~mm}$, the fiber height $h_{f}$ is consider as a first parameter, while the total height of the cell is determined by the fiber volume fraction $c_{f}$, which is the second parameter of this example.

The left edge of the considered unit-cell is fixed while the right edge is free to move horizontally and is subjected to a prescribed rigid body vertical displacement. Periodicity in the vertical direction is imposed by tying the degrees of freedom of the top edge to the corresponding ones of the bottom edge.

The fiber is assumed to deform purely elastically according to constitutive law (24) with $K_{f}=$ $500 / 3 \mathrm{GPa}$ and $G_{f}=100 \mathrm{GPa}$. Regarding the matrix phase, isotropic plasticity according to Section 4 is assumed, combined with the elastic constitutive law (25) with $K_{m}=100 / 3 \mathrm{GPa}$ and $G_{m}=20 \mathrm{GPa}$. 


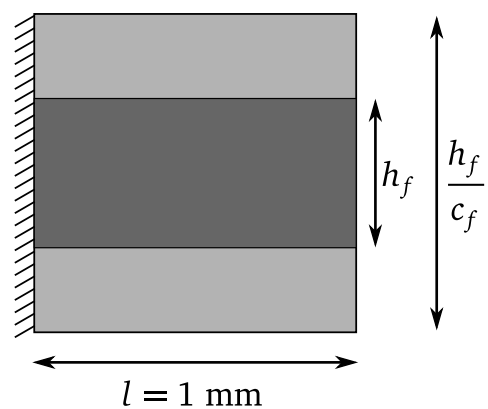

Figure 5: Parametric unit-cell of a fiber composite.

The plastic yield criterion is according to Eq. (28) with initial yield limit $\sigma_{y m}=0.15 \mathrm{GPa}$ and hardening modulus $H_{m}=3.674 \mathrm{GPa}$.

A reference model of this first example is obtained by explicitly discretizing the geometry of Fig. 5 and applying different material properties to the domains covered by the fiber and the matrix. The discretization is based on 12 elements along the length and 5 elements along the height of the fiber. The number of the elements in the matrix material is adapted so that their vertical size will be close to the size of the elements in the fiber but there will never be less than three rows of elements in the matrix on each side of the fiber.

Regarding the homogenized version of this example, the periodicity boundary condition in the vertical direction implies that the homogenized problem is actually one-dimensional. For this reason a single row of the homogenized elements, introduced in the present work, should be sufficient for solving this problem, irrespective of the element height. The homogenized model requires however an additional boundary condition, with respect to the internal kinematic variable $\boldsymbol{d}$. In this example, both components of the $\boldsymbol{d}$ vector field are fixed to zero at both left and right edges of the considered unit-cell.

Fig. 6 shows simulation results for the reference model with an explicitly discretized material micro-structure, for three values of the fiber height $h_{f}=0.1,0.3$ and $0.6 \mathrm{~mm}$ and for four values of the fiber content $c_{f}=0.2,0.4,0.6$ and 0.8 . The provided snapshot corresponds to a final vertical displacement of the right edge equal to $0.2 \mathrm{~mm}$, while the complete simulation is divided into 100 equal size steps. Corresponding results for the homogenized model and the same combinations of the fiber height $h_{f}$ and fiber content $c_{f}$ are shown in Fig. 7. Three rows of elements are presented for the sake of illustration, although the solution is actually independent of the vertical coordinate.

The evolution of the vertical load on the right edge of the considered unit-cell, during all 100 load steps, is shown in Fig. 8, Each diagram compares results from the reference model with the explicitly discretized fiber with results from the homogenized model. At the same time, the solution of the homogenized model without the contribution of the fiber bending stiffness, i.e. with $h_{f}=0$, is also included for comparison. As expected, for any fiber content $c_{f}$, larger fiber height values $h_{f}$ lead to increased shear loads due to the contribution of the fiber bending stiffness. Even for the most slender fiber, with a height to length ratio of $10 \%$, a significant contribution of the fiber bending stiffness can be observed, compared to the curves for $h_{f}=0$ (see first row of diagrams in Fig. 8). In general, the homogenized model delivers results close to the reference model for a wide range of values for $h_{f}$ and $c_{f}$ and for an average shear strain ranging up to 20\%. The importance of accounting for the fiber bending stiffness, through the higher order term introduced in Section 3 , is evident, at least for cases with $h_{f}=0.3$ and $0.6 \mathrm{~mm}$. 


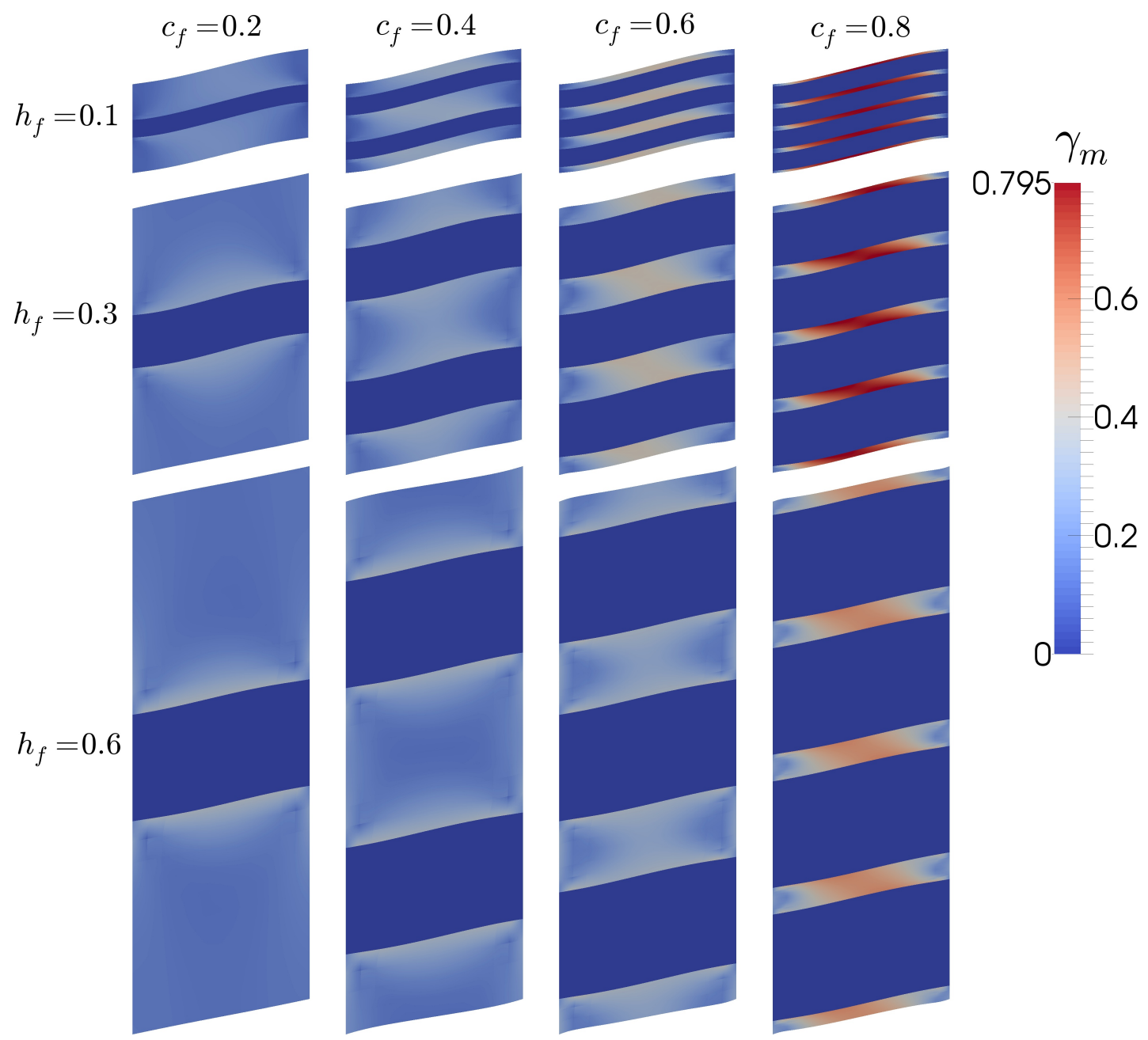

Figure 6: Final simulation step from the reference model of the unit-cell example.

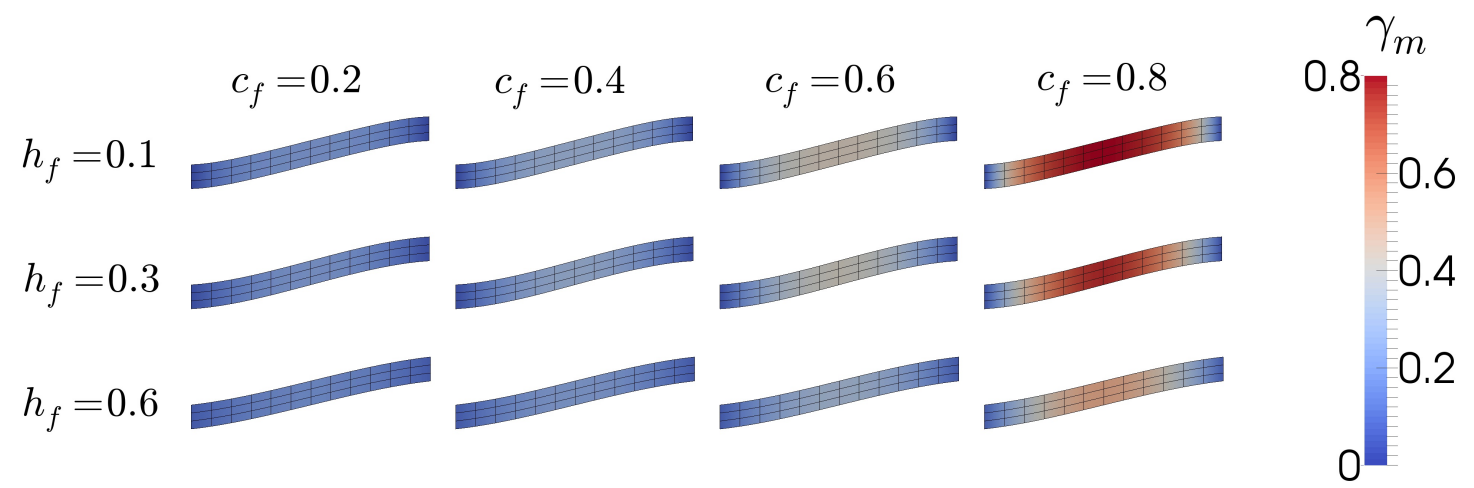

Figure 7: Final simulation step from the homogenized model of the unit-cell example. 

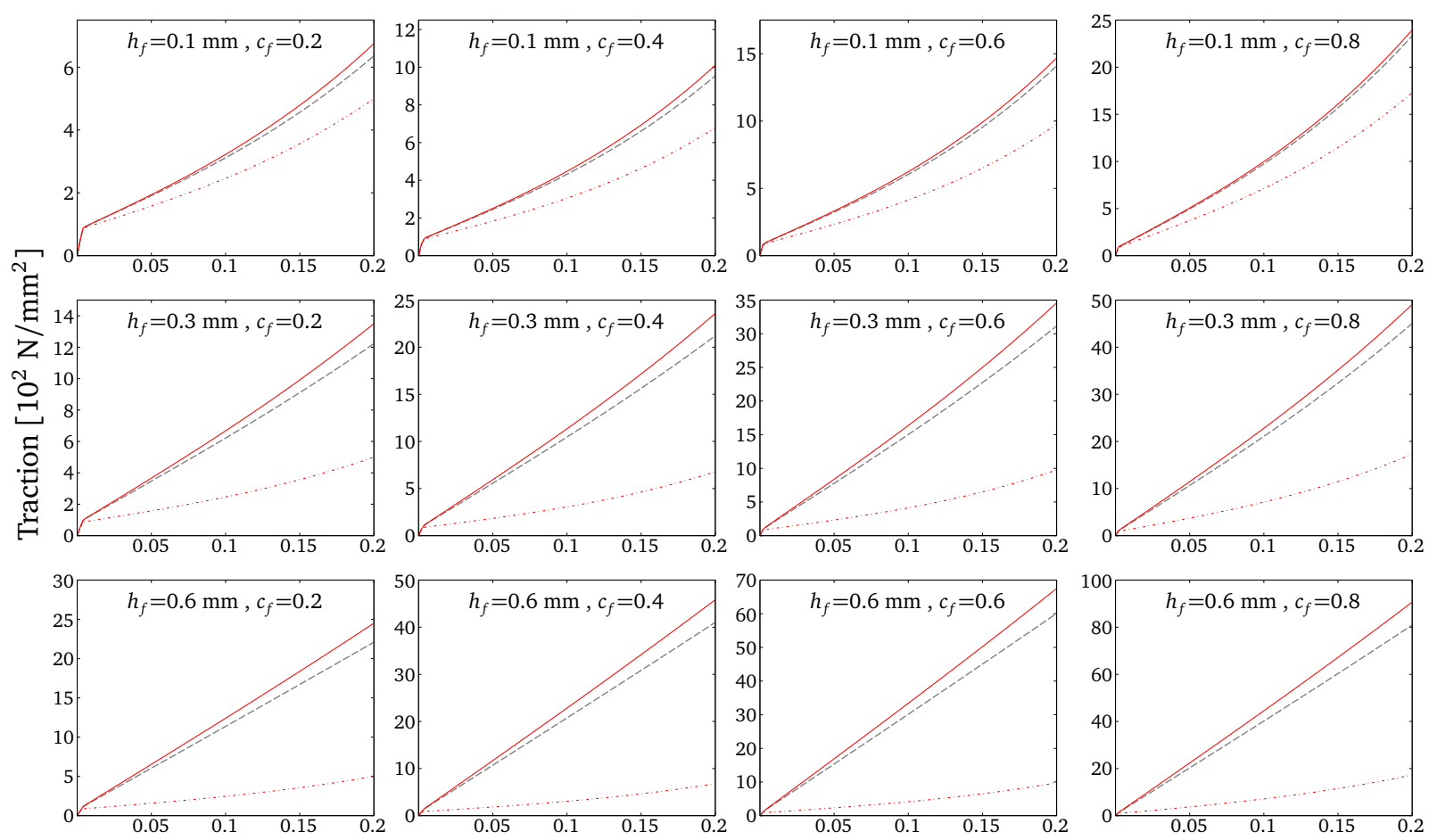

Vertical displacement $[\mathrm{mm}]$

- - - Reference model (discrete)

Homogenized model

- - - Homogenized model without fiber bending stiffness

Figure 8: Evolution of the traction applied to the right edge of the unit-cell as a function of the vertical displacement of the edge for different values of $h_{f}$ and $c_{f}$.

With the intention of being conservative, a relatively fine mesh was used in this example for both the reference and the homogenized model. Nevertheless, satisfactory accuracy can be obtained also with a coarser discretization. The mesh convergence study presented in Fig. 9) shows, for the smallest one of the examined fiber heights, $h_{f}=0.1 \mathrm{~mm}$, that the original mesh can be coarsened by a factor of two without any considerable impact on the calculated vertical traction. Fig. 9) presents only the case of $c_{f}=0.2$, but other fiber content values yield very similar behavior with respect to mesh size. An increased fiber height $h_{f}$ allows even coarser discretization without compromising accuracy. E.g. for the largest one of the examined fiber heights, $h_{f}=0.6 \mathrm{~mm}, 3$ elements along the unit-cell length $l$ deliver results which are indistinguishable from the curves for $h_{f}=0.6 \mathrm{~mm}$, presented in Fig. 8 ,

After recognizing that the optimal discretization length is not directly related to the unit-cell length $l$, but is rather related to the fiber height, a further interpretation of Fig. 9 is possible. In general it can be said that the coarsest of the three meshes with an element size of about $3 \cdot h_{f}$ provides reasonable accuracy.

\subsection{Buckling of a material sample under compression}

Studying micro-buckling in fiber reinforced composite materials is a major motivation for developing the here presented model. In this section, an example of a composite material sample under compression demonstrates the capability of the proposed model to predict micro-buckling initiation with reasonable accuracy. 


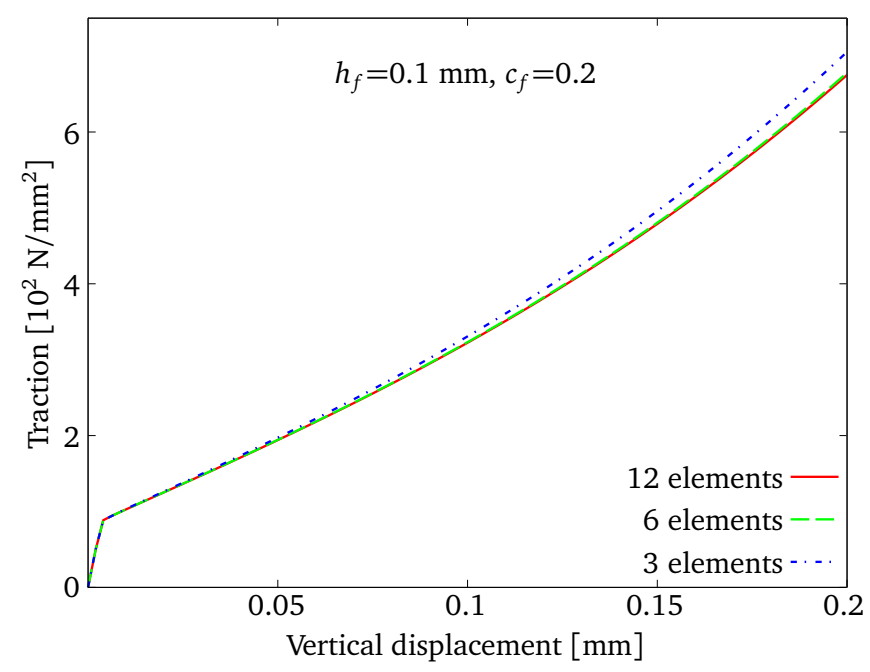

Figure 9: Evolution of the traction applied to the right edge of the unit-cell for $h_{f}=0.1 \mathrm{~mm}, c_{f}=0.2$ and different numbers of elements along its length $l$.

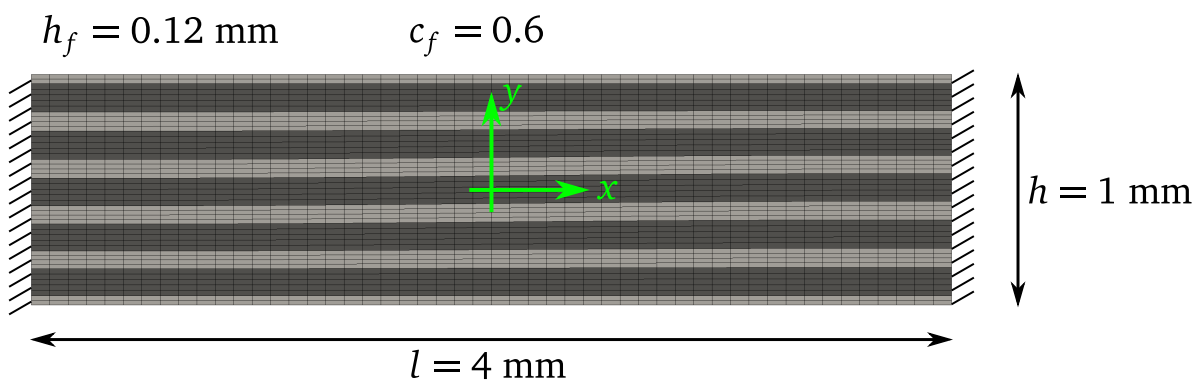

Figure 10: Basic geometry of a fiber composite sample subjected to uniaxial compression.

Fig. 10 shows the undeformed configuration of a reference structure with five explicitly defined fibers. Both fibers and the matrix are discretized with 8-node second order quadrilateral elements. There are 50 elements along the length of the sample, five elements in each layer corresponding to a fiber and 4 elements in each matrix layer. On top of the theoretically parallel and equidistant arrangement, an additional waviness error is applied to the fibers by transforming each point $(x, y)$ to $(x, y+\delta y)$, where

$$
\delta y(x, y)=\frac{a_{w}}{2} \max \left(1-\left(\frac{2 y}{h_{w}}\right)^{2}, 0\right) \sin \left(\frac{\pi}{2} \max \left(\min \left(\frac{2 x}{l_{w}}, 1\right),-1\right)\right),
$$

with $a_{w}=0.02 \mathrm{~mm}$ and $h_{w}=l_{w}=0.8 \mathrm{~mm}$. Transformation (57) means that the middle fiber exhibits a waviness amplitude $a_{w}$, while the waviness of the other fibers decades with increasing distance from the middle one. For the chosen value of $h_{w}$, the top and bottom fibers of Fig. 10 are practically free of any waviness.

As in the previous example, the fibers are elastic with $K_{f}=500 / 3 \mathrm{GPa}$ and $G_{f}=100 \mathrm{GPa}$. In this example, the matrix material is assumed to be two orders of magnitude more flexible than the fiber, with $K_{m}=5 / 3 \mathrm{GPa}$ and $G_{m}=1 \mathrm{GPa}$. The matrix material can also deform plastically with initial yield limit $\sigma_{y m}=4.5 \cdot 10^{-3} \mathrm{GPa}$ and linear hardening modulus $H_{m}=0.1225 \mathrm{GPa}$. 
The corresponding homogenized model is discretized with 20 by 10 elements in the length and height directions respectively. Instead of the geometrical transformation (57), an initial fiber waviness needs to be defined in the homogenized model in terms of the orientation angle $\varphi_{0}(x, y)$, which is obtained through differentiation of Eq. (57), as

$$
\varphi_{0}(x, y)=\arctan \left(\frac{\pi}{l_{w}} \frac{a_{w}}{2} \max \left(1-\left(\frac{2 y}{h_{w}}\right)^{2}, 0\right) \cos \left(\frac{\pi}{2} \max \left(\min \left(\frac{2 x}{l_{w}}, 1\right),-1\right)\right)\right) .
$$

Fig. 11 shows the evolution of the horizontal and vertical reaction forces as the imposed axial compression increases from 0 to 5\%, corresponding to a reduction of the distance between the supported ends from 4 to $3.8 \mathrm{~mm}$.

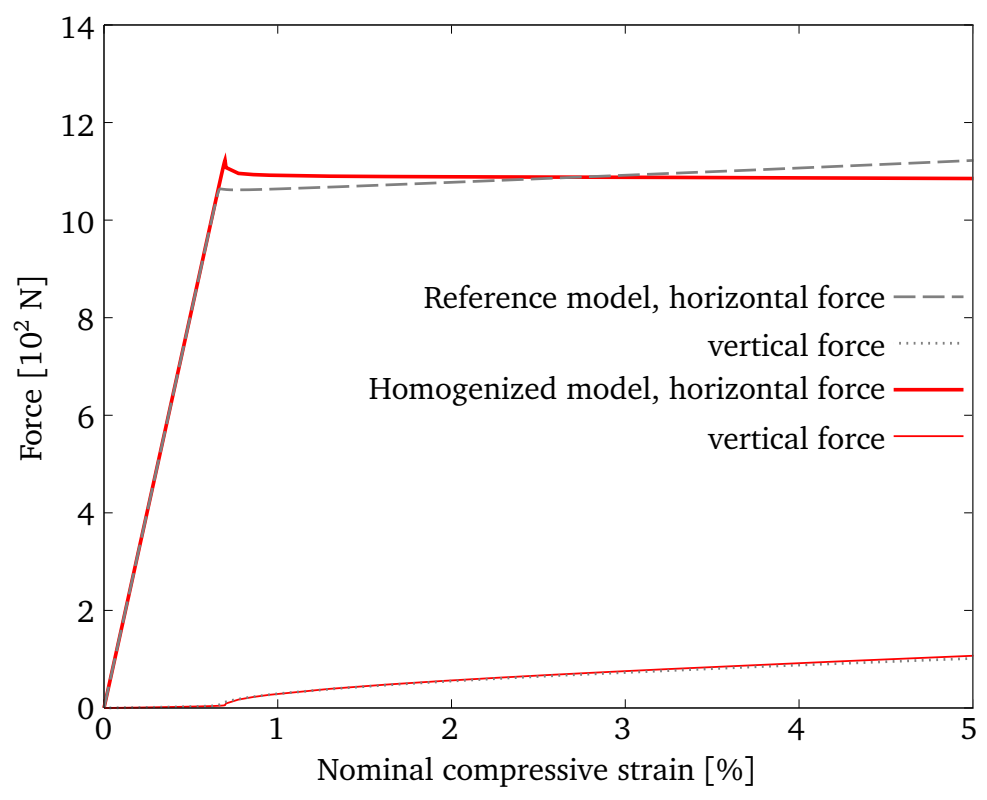

Figure 11: Evolution of reaction forces during compression of a sample with 5 fibers of $h_{f}=0.12 \mathrm{~mm}$.

In order to demonstrate how the proposed homogenization accounts for the fiber bending stiffness, a second variant of the considered example was solved. In this variant all further parameters were kept unchanged except for the fiber height, which was halved to $h_{f}=0.06 \mathrm{~mm}$, and the number of fibers in the reference model, which was doubled from 5 to 10, in order to maintain the original fiber content. The results presented in Fig. 12) show that the homogenized model can capture the reduction in the buckling initiation force, due to the reduced fiber bending stiffness, quite accurately. In the post-buckling regime, for both fiber heights of 0.06 and $0.12 \mathrm{~mm}$, the homogenized model predicts a rather constant compressive force, while the reference model shows a slight increase of the horizontal force component with increasing compressive strain. This discrepancy has to be attributed to the simplifications made in the homogenized model, i.e. the neglecting of higher order terms in the matrix material and the approximate nature of Eq. (45). All results regarding buckling initiation points in Figs. 11 and 12 are summarized in Table 1 .

Fig. 13 shows the spatial distribution of the accumulated plastic strain in the matrix material corresponding to the buckling initiation point of the curves depicted in Fig. 12. The homogenized version seems to capture the considered field reasonably well. A more quantitative comparison is available in Fig. 14, which shows the evolution of the accumulated plastic strain in the matrix material 


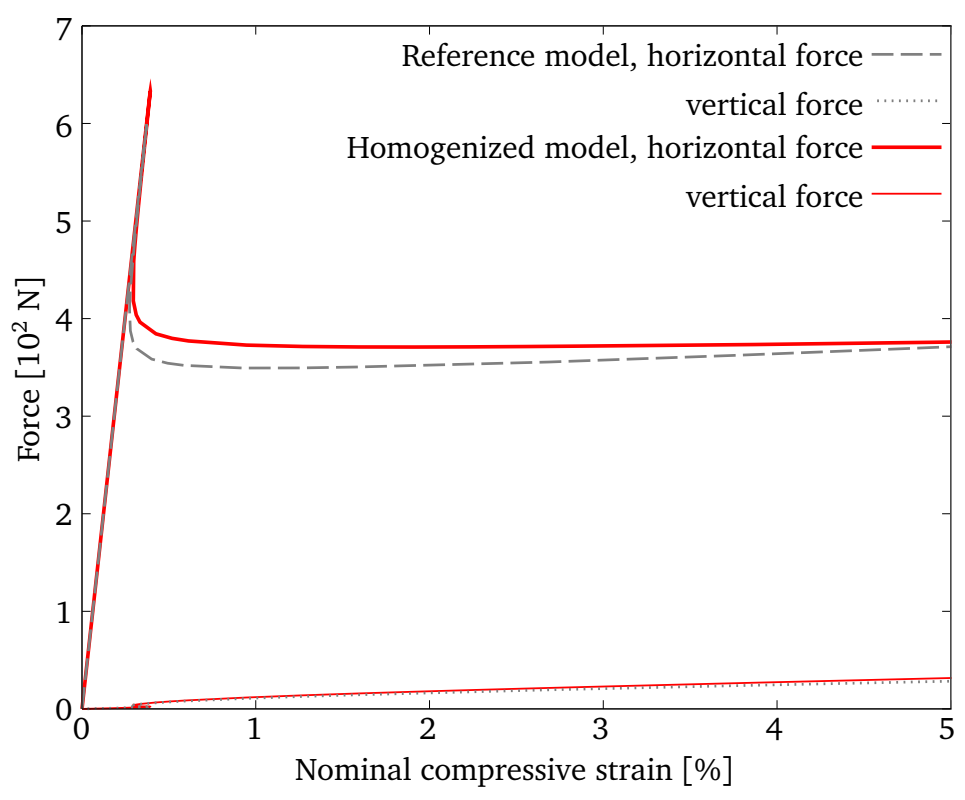

Figure 12: Evolution of reaction forces during compression of a sample with 10 fibers of $h_{f}=0.06 \mathrm{~mm}$.

Table 1: Summary of calculated buckling initiation points for the second example.

\begin{tabular}{lcc} 
& Compressive strain & Horizontal load \\
\hline Reference model with 5 individual fibers & $6.70 \%$ & $1064.0 \mathrm{~N}$ \\
Homogenized model with 5 fibers & $6.96 \%$ & $1122.9 \mathrm{~N}$ \\
\hline Reference model with 10 individual fibers & $3.72 \%$ & $599.0 \mathrm{~N}$ \\
Homogenized model with 10 fibers & $3.93 \%$ & $633.4 \mathrm{~N}$ \\
\hline
\end{tabular}

at the center of the sample subjected to compression. Up to a nominal compression of $1 \%$ the results from the two models are practically indistinguishable, while reasonable agreement between the two models is maintained until the final compression ratio of $5 \%$. This comparison demonstrates that the homogenized model is capable of capturing the micro-mechanical behavior of the simulated composite very precisely.

It is important to note, that applying Newton's algorithm in its standard form as outlined in Section 7 is not sufficient for solving this second example. Especially in case of the results shown in Fig. 12, it becomes obvious that a numerical continuation algorithm is necessary for tracking the unstable branch following the buckling initiation. The here presented results rely on the arc-length based algorithm described in [21]. The applied numerical continuation algorithm includes variable load stepping which is crucial for determining the buckling initiation point accurately, without requiring an excessive number of load steps for completing the simulation. For the case presented in Fig. 11 . 46 and 50 load steps were required for the reference and the homogenized model respectively, while 52 and 72 load steps were necessary for each of the two models presented in Fig. 12.

Although the default mesh used in the homogenized model of this example, depicted in Fig. 13 , was chosen according to the conclusions from the mesh convergence study of the first example, some 


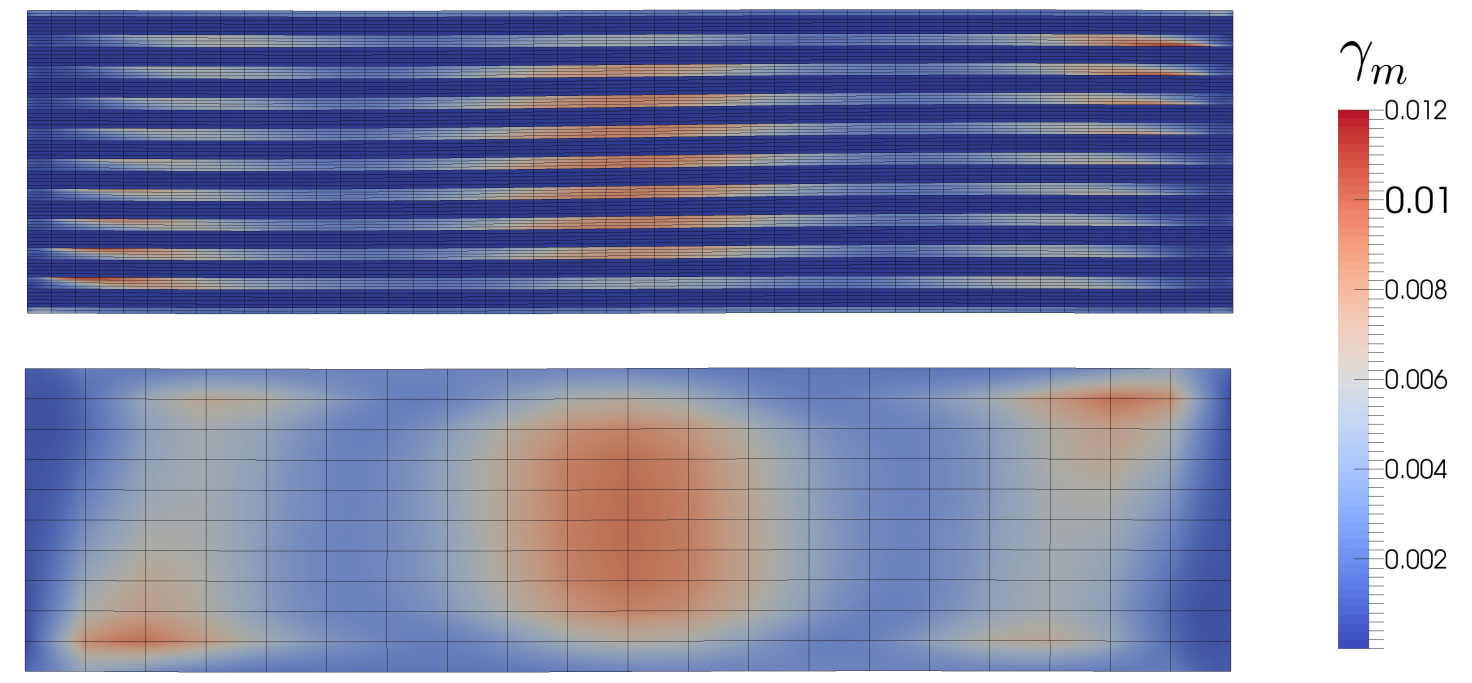

Figure 13: Deformed configuration and accumulated plastic strain in the matrix material at buckling initiation for the second example variant with 10 reinforcing fibers, for the reference and the homogenized models (top and bottom respectively).

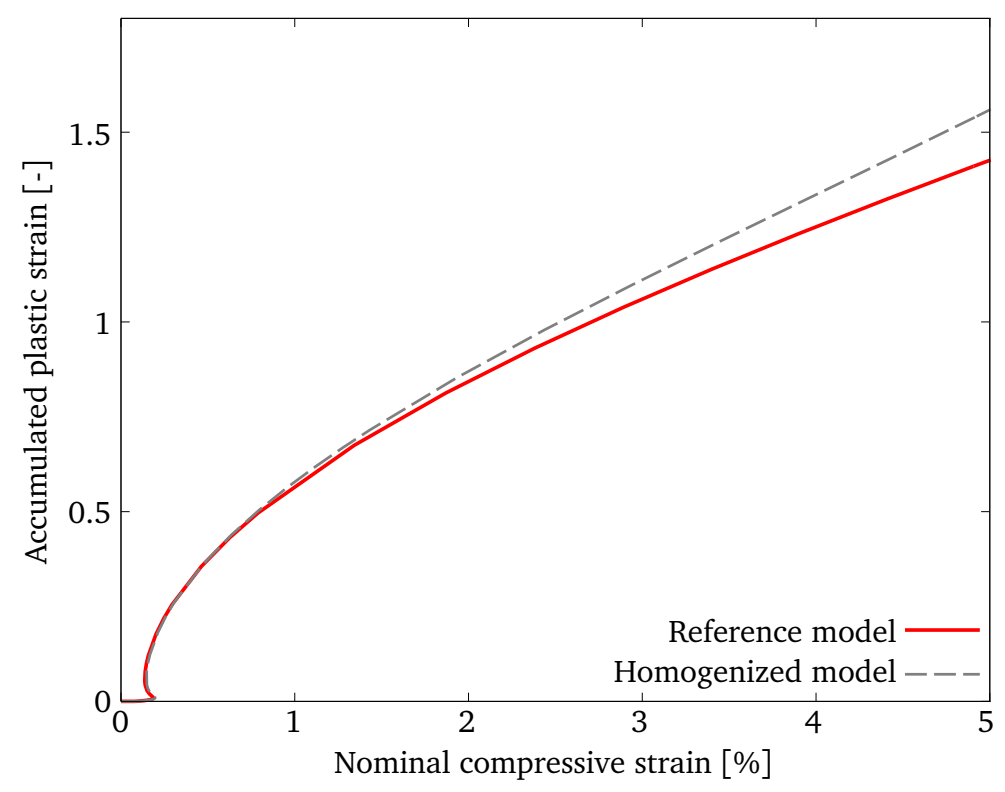

Figure 14: Evolution of the accumulated plastic strain at the center of a sample with 10 fibers of $h_{f}=0.06 \mathrm{~mm}$ as a function of the nominal compressive strain.

further investigations were carried out in the context of the present example. Fig. 15 shows how the homogenized model results from Fig. 12 (red curve) are affected by a coarsening and refinement of the original mesh by a factor of two. The coarsened mesh delivers considerably different results especially in the post-buckling response but also to a smaller extent with respect to the buckling initiation point. The refined mesh provides very similar results to the original mesh. Hence it appears that the initial discretization size was actually a successful choice. 

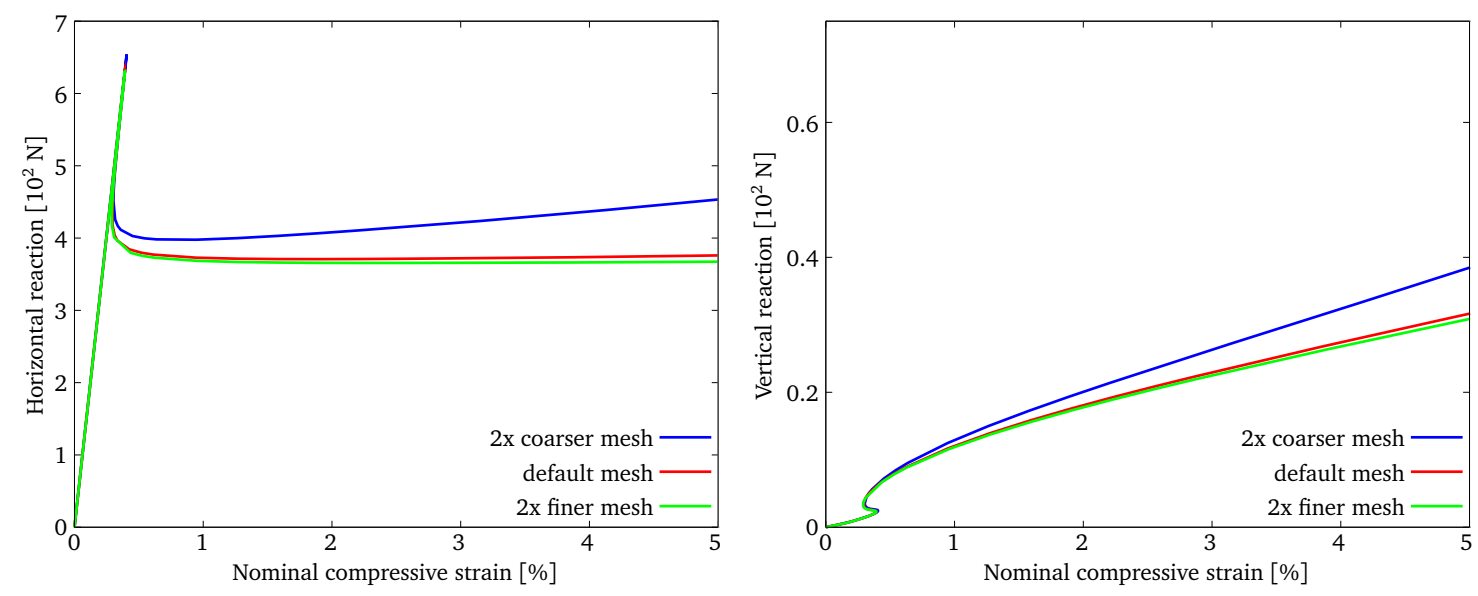

Figure 15: Evolution of reaction forces during compression of a homogenized sample with $h_{f}=0.06 \mathrm{~mm}$ at different mesh sizes.
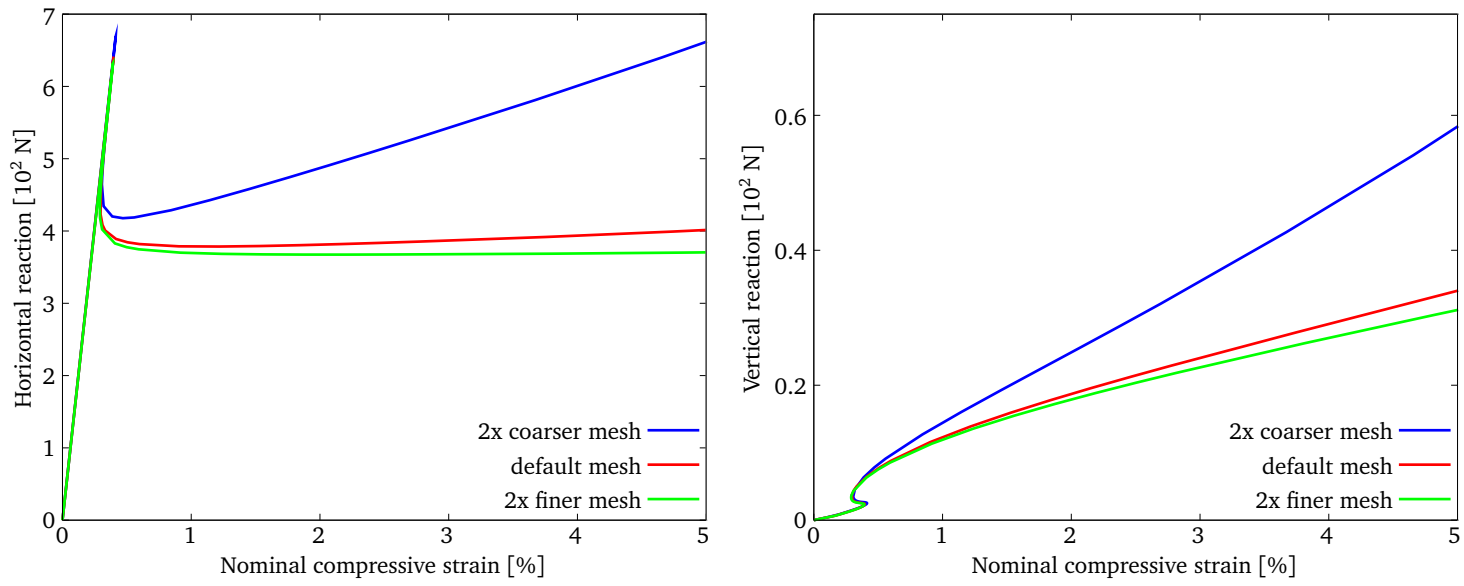

Figure 16: Evolution of reaction forces during compression of a homogenized sample with $h_{f}=0.06 \mathrm{~mm}$ at different mesh sizes and with a linear (4-node element) approximation of $\boldsymbol{d}$ instead of a second order one (8-node element).

Fig. 16 presents a similar mesh convergence study with a different finite-element approximation of the unknown $\boldsymbol{d}$. Instead of the original second order approximation, a linear one is employed, based on 4-node quadrilateral elements. It should be underlined that the approximation of the displacement field $u$ remains unchanged, still based on 8-node quadrilateral elements. The results in Fig. 16 show a stronger mesh size dependence than in Fig. 16 and actually suggest that the refined mesh is necessary in order to achieve similar accuracy, like with the default mesh and the second order approximation of $\boldsymbol{d}$. 


\section{Concluding remarks}

A numerical method has been proposed for the homogenization of unidirectionally reinforced fiber composite materials under plane-strain conditions, accounting for the fiber bending stiffness. Emphasis was put on deriving a homogenization method directly from the fundamental continuum mechanics of the composite material micro-structure. An important observation was made, that standard homogenization leads to deformation tensors for the individual constituents which do not necessarily fulfill the deformation gradient compatibility condition. Motivated by this observation, a technique for recovering the possibly violated compatibility condition within a single material layer was proposed here and applied to the fiber material, resulting in a higher order term, which as expected accounts for the fiber bending stiffness and thereto related size effects.

A series of numerical examples demonstrated how the proposed method compares to an explicit discretization of the fiber and matrix phases in a composite material. The first example, employing pure shearing of a periodic structure, in direction transverse to the fiber axis, showed that the bending stiffness of fibers with a diameter even as low as $10 \%$ of the width of the shear zone, still has a considerable impact on the observed displacement versus force response. In general, the homogenized model was capable of capturing the evolution of the shear force with deviations from the reference model, with the explicitly discretized micro-structure, not exceeding the level of $10 \%$. The second example showed that the homogenized model is capable of predicting a fiber diameter dependent buckling initiation with good accuracy. For both fiber diameters studied in this example, the buckling point predicted by the homogenized model did not deviate more than $6 \%$ from the reference model.

Extending the proposed homogenization procedure to cases beyond the here assumed plane-strain condition and accounting for variations through the matrix layer thickness are goals reserved for future work. Modeling of fiber breakage and the application of the model to experimentally validated cases are further objectives.

\section{Acknowledgements}

This work was supported by the Danish Council for Independent Research, through the Sapere Aude project "Higher Order Theories in Solid Mechanics".

\section{Appendix A: Derivation of the kinematic decomposition}

With vectors $\boldsymbol{T}$ and $\boldsymbol{N}$ being orthonormal, the following identity holds

$$
T T^{T}+N N^{T}=I .
$$

Eq. (5) multiplied by $\boldsymbol{T}^{T}$ from the right becomes

$$
\boldsymbol{F}_{u} \boldsymbol{T} \boldsymbol{T}^{T}=\boldsymbol{F}_{m} \boldsymbol{T} \boldsymbol{T}^{T}=\boldsymbol{F}_{f} \boldsymbol{T} \boldsymbol{T}^{T},
$$

while Eq. (7) multiplied by $\boldsymbol{N}^{T}$ from the right, after some reordering of terms yields

$$
\begin{aligned}
& \boldsymbol{F}_{m} \boldsymbol{N} \boldsymbol{N}^{T}=\boldsymbol{F}_{u} \boldsymbol{N} \boldsymbol{N}^{T}+\frac{1}{c_{m}} \boldsymbol{d} \boldsymbol{N}^{T} \\
& \boldsymbol{F}_{f} \boldsymbol{N} \boldsymbol{N}^{T}=\boldsymbol{F}_{u} \boldsymbol{N} \boldsymbol{N}^{T}-\frac{1}{c_{f}} \boldsymbol{d} \boldsymbol{N}^{T} .
\end{aligned}
$$

Summation of Eqs. (61) with Eqs. (60) and exploiting identity (59), proves Eqs. (8) and (9). 


\section{Appendix B: Enhanced deformation tensor compatibility}

Below, it is shown that the enhanced deformation tensor $\tilde{\boldsymbol{F}}_{f}$ defined through Eqs. (14) and (9), fulfills a compatibility condition in the sense of Eq. (10). For this purpose, it is necessary to state the derivatives

$$
\frac{\partial \chi_{N}}{\partial X_{1}}=N_{1} \text { and } \frac{\partial \chi_{N}}{\partial X_{2}}=N_{2}
$$

and also notice in Fig. 1 that

$$
T_{1}=N_{2} \text { and } T_{2}=-N_{1} .
$$

The last two equations are then used for showing that the spatial gradients of $\tilde{\boldsymbol{F}}_{f}$ from Eq. (14), evaluated at $\chi_{N}=0$, fulfill the following condition

$$
\begin{aligned}
\frac{\partial \tilde{F}_{f i 2}}{\partial X_{1}}-\frac{\partial \tilde{F}_{f i 1}}{\partial X_{2}}= & \frac{\partial F_{u i 2}}{\partial X_{1}} \frac{\partial F_{u i T}}{\partial X_{2}} 0 \\
& -\frac{1}{c_{f}}\left(\frac{\partial d_{i}}{\partial X_{1}} N_{2}-\frac{\partial d_{i}}{\partial X_{2}} N_{1}+\left(\frac{\partial N_{2}}{\partial X_{1}}-\frac{\partial N_{1}}{\partial X_{2}}\right) d_{i}\right) \\
& -\frac{\partial \chi_{N}}{\partial X_{1}} \frac{1}{c_{f}}\left(\nabla \boldsymbol{d} \boldsymbol{T} \boldsymbol{T}^{T}+\operatorname{div} \boldsymbol{T} \boldsymbol{d} \boldsymbol{T}^{T}\right)_{i 2} \\
& +\frac{\partial \chi_{N}}{\partial X_{2}} \frac{1}{c_{f}}\left(\nabla \boldsymbol{d} \boldsymbol{T} \boldsymbol{T}^{T}+\operatorname{div} \boldsymbol{T} \boldsymbol{d} \boldsymbol{T}^{T}\right)_{i 1} \\
= & -\frac{1}{c_{f}}\left(\frac{\partial d_{i}}{\partial X_{1}} N_{2}-\frac{\partial d_{i}}{\partial X_{2}} N_{1}+\operatorname{div} \boldsymbol{T} d_{i}\right) \\
& -N_{1} \frac{1}{c_{f}}(\nabla \boldsymbol{d} \boldsymbol{T}+\operatorname{div} \boldsymbol{T} \boldsymbol{d})_{i} T_{2} \\
& +N_{2} \frac{1}{c_{f}}(\nabla \boldsymbol{d} \boldsymbol{T}+\operatorname{div} \boldsymbol{T} \boldsymbol{d})_{i} T_{1} \\
= & -\frac{1}{c_{f}}\left(\nabla \boldsymbol{d}\left(\begin{array}{c}
N_{2} \\
-N_{1}
\end{array}\right)+\operatorname{div} \boldsymbol{T} \boldsymbol{d}\right)_{i} \\
& -\frac{1}{c_{f}}\left(N_{1} T_{2}-N_{2} T_{1}\right)(\nabla \boldsymbol{d} \boldsymbol{T}+\operatorname{div} \boldsymbol{T} \boldsymbol{d})_{i} \\
= & -\frac{1}{c_{f}}\left(1-N_{1} T_{2}-\widehat{\left.N_{2} T_{1}\right)(\nabla \boldsymbol{d} \boldsymbol{T}+\operatorname{div} \boldsymbol{T} \boldsymbol{d})_{i}=0},\right.
\end{aligned}
$$

for $i=1,2$, which proves that $\tilde{\boldsymbol{F}}_{f}$ is compatible in the sense of Eq. 10 . The proof for $\tilde{\boldsymbol{F}}_{m}$ from Eq. (13) is completely equivalent and it is therefore omitted.

\section{Appendix C: Simplification of the plastic flow rule equation}

According to Eqs. (25) and (19), the deviatoric part of the Kirchhoff stress tensor is

$$
\operatorname{dev}(\boldsymbol{\tau})=G|\boldsymbol{F}|^{-2 / 3}\left(\operatorname{dev}\left(\ln \boldsymbol{b}^{e, t r i a l}\right)-2 \boldsymbol{\Delta} \boldsymbol{\epsilon}^{p}\right),
$$


where $\boldsymbol{\Delta} \boldsymbol{\epsilon}^{p}$ is according to Eq. (31) intrinsically deviatoric.

Substitution of Eq. (65) into Eq. (31) yields

$$
\operatorname{dev}\left(\ln \boldsymbol{b}^{e, \text { trial }}\right)=\left(\frac{\|\operatorname{dev}(\boldsymbol{\tau})\||\boldsymbol{F}|^{2 / 3}}{\left\|\boldsymbol{\Delta} \boldsymbol{\epsilon}^{p}\right\| G}+2\right) \boldsymbol{\Delta} \boldsymbol{\epsilon}^{p},
$$

which essentially proves Eqs. (32) and (33).

\section{References}

[1] B. Budiansky and N. Fleck, "Compressive failure of fiber composites," Journal Of The Mechanics And Physics Of Solids, vol. 41, no. 1, pp. 183-211, 1993.

[2] S. Kyriakides, E. J. Perry, and K. M. Liechti, "Instability and failure of fiber composites in compression," Applied Mechanics Reviews, vol. 47, no. 6, pp. S262-S268, 1994.

[3] N. Fleck and J. Shu, "Microbuckle initiation in fibre composites: a finite element study," Journal of the Mechanics and Physics of Solids, vol. 43, no. 12, pp. 1887-1918, 1995.

[4] M. Sutcliffe, N. Fleck, and X. Xin, "Prediction of compressive toughness for fibre composites," Proceedings of the Royal Society A - Mathematical Physical and Engineering Sciences, vol. 452, no. 1954, pp. 2443-2465, 1996.

[5] N. Fleck, "Compressive failure of fiber composites," Advances in Applied Mechanics, vol. 33, pp. 43-117, 1997.

[6] J. Christoffersen and H. M. Jensen, "Kink band analysis accounting for the microstructure of fiber reinforced materials," Mechanics of Materials, vol. 24, no. 4, pp. 305-315, 1996.

[7] M. Romanowicz, "Initiation of kink bands from regions of higher misalignment in carbon fiberreinforced polymers," Journal of Composite Materials, vol. 48, no. 19, pp. 2387-2399, 2014.

[8] J. L. Wind, S. Steffensen, and H. M. Jensen, "Comparison of a composite model and an individually fiber and matrix discretized model for kink band formation," International Journal of Non-linear Mechanics, vol. 67, pp. 319-325, 2014.

[9] R. Hill, The Mathematical Theory of Plasticity. 1950.

[10] F. J. Montáns and K.-J. Bathe, "Large strain anisotropic plasticity including effects of plastic spin," 3rd M.I.T. Conference on Computational Fluid and Solid Mechanics, pp. 377-381, 2005.

[11] C. Sansour, I. Karšaj, and J. Sorić, "A formulation of anisotropic continuum elastoplasticity at finite strains. Part I: Modelling," International Journal of Plasticity, vol. 22, no. 12, pp. 23462365, 2006.

[12] A. Seitz, A. Popp, and W. A. Wall, "A semi-smooth newton method for orthotropic plasticity and frictional contact at finite strains," Computer Methods in Applied Mechanics and Engineering, vol. 285, pp. $228-254,2015$.

[13] E. Cosserat and F. Cosserat, Théorie des corps déformables. A. Hermann et Fils, 1909. 
[14] R. Sievert, "A geometrically nonlinear elasto-viscoplasticity theory of second grade," Technische Mechanik, vol. 31, no. 1-2, pp. 83-111, 2011.

[15] S. Nemat-Nasser, "Averaging theorems in finite deformation plasticity," Mechanics of Materials, vol. 31, no. 8, pp. $493-523,1999$.

[16] A. Eterovic and K.-J. Bathe, "A hyperelastic-based large strain elastoplastic constitutive formulation with combnined isotropic-kinematic hardening using the logarithmic stress and strain measures," International Journal for Numerical Methods in Engineering, vol. 30, no. 6, pp. 10991114, 1990.

[17] J. Simo, "Algorithms for static and dynamic multiplicative plasticity that preserve the classical return mapping schemes of the infinitesimal theory," Computer Methods in Applied Mechanics and Engineering, vol. 99, no. 1, pp. 61-112, 1992.

[18] L. Brančík, "Techniques of matrix exponential function derivative for electrical engineering simulations," Proceedings of the IEEE International Conference on Industrial Technology, pp. 26082613, 2006.

[19] N. Higham, Functions of Matrices: Theory and Computation. Society for Industrial and Applied Mathematics, SIAM, 2008.

[20] J. Pommier and Y. Renard, "GetFEM++, an open source generic C++ library for finite element methods." http://download.gna.org/getfem/html/homepage/.

[21] T. Ligursky and Y. Renard, "A continuation problem for computing solutions of discretised evolution problems with application to plane quasi-static contact problems with friction," Computer Methods in Applied Mechanics and Engineering, vol. 280, pp. 222-262, 2014. 